\title{
Phaseless Spherical Near-Field to Far-Field Transformation Algorithm via Sparsity of Spherical Mode Coefficients
}

\author{
Jiaqi Wang $\left(D\right.$, Yinghong Wen, Dan Zhang ${ }^{D}$, and Jinbao Zhang \\ Electromagnetic Compatibility Laboratory, Beijing Jiaotong University, Beijing, China \\ Correspondence should be addressed to Dan Zhang; zhang.dan@bjtu.edu.cn
}

Received 25 October 2021; Accepted 28 December 2021; Published 7 March 2022

Academic Editor: Shah Nawaz Burokur

Copyright ( $\odot 2022$ Jiaqi Wang et al. This is an open access article distributed under the Creative Commons Attribution License, which permits unrestricted use, distribution, and reproduction in any medium, provided the original work is properly cited.

In order to overcome the difficulty in obtaining accurate phase information in antenna near-field measurement, this study proposes a phase recovery algorithm that can be applied to phaseless spherical near-field sampling data. Based on the sparsity of spherical mode coefficients (SMCs), under the condition of unknown sparsity level and low oversampling rate, the proposed algorithm can accurately recover SMC to obtain high-precision antenna radiation characteristics. To improve the recovery performance of the algorithm, a spherical sampling strategy matching with the algorithm is also presented. By calculating the correlation of the measurement matrix and comparing the accuracy of the reconstructed far field, a set of optimal parameter settings including the number of spheres, the distribution of the measurement points, radius difference, and polarization mode is determined. On the basis of the obtained measurement data, the performance advantage of the presented algorithm in recovering SMC is demonstrated.

\section{Introduction}

Antenna near-field measurements have many advantages, including being able to be carried out in a relatively small anechoic chamber and low measurement cost [1]. With the high-performance transformation algorithm, accurate antenna far-field data can be obtained [2, 3]. In particular, the spherical near-field measurement can provide the complete three-dimensional radiation characteristics of antenna under test (AUT), and the reconstructed far field has the highest precision. However, the accurate characterization of phase at high frequencies in spherical nearfield measurement is challenging. Due to the cable movement, the drift of measuring instruments, and the deviation of positioning equipment, the phase information is extremely unstable or even unavailable, resulting in large errors in the far-field extrapolation. In addition, in over-the-air (OTA) tests, the phase reference of the active device is difficult to extract or accurately transmit to the vector network analyzer. Therefore, the antenna community has been committed to phaseless near-field measurement technology.
The existing spherical phaseless near-field measurement techniques can be broadly classified into three categories. Among them, holography [4] and interference technology [5] have lower postprocessing difficulty but need to use specially calibrated measurement equipment. In addition to the above two schemes, modeling the solution of SMC as a phase recovery problem provides a new idea for the subsequent near-field-far-field transformation (NFFFT). In [6-8], an iterative algorithm is proposed to enable two concentric measuring spheres to continuously exchange information until convergence. However, a large number of near-field data need to be acquired to guarantee high convergence accuracy. In [9], the authors directly solve the SMC using Gerchberg-Saxton, Wirtinger flow, PhaseLift, PhaseLamp, and PhaseMax, to perform an approximate reconstruction of the far field when the oversampling rate is 3. In addition to the improved transform algorithm, different sampling strategies are used to add extra information and reduce the solution space so as to overcome the information loss inherent in phaseless measurement. In $[5,10]$, the author restores SMC by inserting the linear combination amplitude of the probe into PhaseCut and Wirtinger flow. In 
the case of 5 times oversampling, the success rate of the farfield reconstruction is up to $90 \%$. In [11], the author scans the AUT twice on sphere and plane, respectively. When combined with Wirtinger flow, the extrapolated far-field error can be reduced to $-35 \mathrm{~dB}$ at 3.57 times oversampling. In [12], multiple types of sampling surfaces are investigated. The numerical results indicate that polyhedrons exhibit more stable reconstruction performance than planes. Moreover, the combination of sphere and tetrahedron demonstrates the most robust relationship to the radius variation.

In order to continue reducing the required near-field sampling points, in [9], the author utilizes the prior information on the sparsity of SMC. Three sparse phase recovery algorithms, namely, CPRL, CoPRAM, and SPARTA, are applied to solve SMC to reconstruct the far field. Numerical results reveal that the three algorithms have low recovery success rates and large errors in the extrapolated far field. Even though the above algorithm has been proved in [13-15] to have good performance in recovering Gaussian data with sufficiently low sparsity level, it is intrinsically not suitable for spherical NFFFT problem. CPRL [13] is based on the lifting idea, resulting in slow operation and extremely high memory occupancy when restoring longer SMC vectors. CoPRAM [15] and SPARTA [14] devote themselves to the recovery of real signals yet ineffective for complex vectors. In addition, both require that the quantity of nonzero elements of the vector to be estimated is known and the sparsity level is sufficiently low. As a complex vector with an unknown sparse level, SMC does not conform to the above properties. Furthermore, in the subsequent iterations, the atoms discarded by CoPRAM and the gradient truncated by SPARTA contain important information, leading to lower recovery accuracy of SMC. In summary, the three algorithms are not applicable to the spherical NFFFT problem.

In conclusion, the problem that SMC cannot be successfully derived at low sampling rates remains to be solved. Therefore, a phase recovery algorithm adapted to phaseless spherical near-field measurement needs to be investigated, which can accurately recover SMC under the conditions of uncertain sparsity level and low oversampling rate.

In this study, a phase recovery algorithm for the spherical NFFFT problem is proposed, which can accurately recover SMC at a low oversampling rate, a sparse complex vector with an uncertain sparsity level. The algorithm employs $\ell_{1}$-norm to guarantee the sparsity of the signal. By reducing the dimension of a complex model to a real number, the signal sparsity level is decreased, and thus, the recovery capability is improved as well. Aiming at the problem that the sparsity level of the vector to be solved is unidentified, the algorithm sets the sparse regularization parameter to decrease from a larger value, with priority given to satisfying the sparsity of the solution in the early stage of the iteration. As the weight of the sparse regularization term is adjusted downward, the error between the recovery and the measured amplitude can be continuously reduced. To be applicable for the recovery of complex signals, the algorithm calculates the gradient of the objective function to the real and imaginary parts of the unknown vector, respectively. In addition, the Barzilai-Borwein step [16] is adopted to enhance the convergence performance. Meanwhile, in order to improve the recovery accuracy of the algorithm, the optimal spherical sampling strategy is designed by calculating the correlation of the measurement matrix [17] and comparing the precision of the AUT's radiation pattern. The algorithm still exhibits strong performance in recovering SMC at a low oversampling rate.

This study is organized as follows. Section 2 mainly establishes the mathematical model of the phaseless spherical NFFFT and outlines the research method of the study. Section 3 begins with an introduction of the improved phase recovery algorithm, including the overall framework, the objective function, and the solution process of the suboptimization problem. The recovery performance for Gaussian data is also demonstrated in this section. Furthermore, Section 3 investigates the settings of parameters including the number of spheres, the distribution of measurement points, radius difference, and the polarization mode, so as to determine an effective sampling strategy. With the measurement data acquired by the above sampling method, the strong ability of the algorithm in recovering SMC is demonstrated in Section 4.

\section{System Model}

As depicted in Figure 1, the spherical near-field measurement can be divided into two steps: sampling and near-field to far-field transformation. The core of the transformation procedure lies in the solution of SMC. When the phase information is missing, the solution process can be modeled as the phase recovery problem:

$$
\begin{array}{r}
\text { find } T, \\
\text { subject to }|w|=|\Psi T|,
\end{array}
$$

where $|w| \in \mathbb{R}^{M}$ represents the measured amplitude vector, and $\psi \in \mathbb{C}^{M \times N}$ is the measurement matrix containing the Wigner $D$ function and the probe response constant, both of which are constructed according to the information of the near-field sampling points. $T \in \mathbb{C}^{N}$ represents the SMC to be calculated. $T_{1}$ and $T_{2}$ correspond to TE and TM modes, respectively. The total number of spherical modes is given by $N=2 B(B+2)=2 B^{2}+4 B$, where $B$ denotes the band limit constant of AUT.

However, the radiation characteristics of AUT can be precisely described by adopting $p$ of the spherical modes, and $p \ll N$. In particular, the sparsity of SMC is more significant when the center of the sampling sphere coincides with the AUT's phase center and/or AUT exhibits geometric symmetry [18-20].

As discussed in Section 1, when the oversampling rate is low, (1) cannot be successfully solved to derive the exact SMC. To tackle this problem, the algorithm proposed in this study makes full use of the sparsity of SMC, imposes sparsity constraints on the vector to be estimated, reduces the solution space, and seeks the sparest SMC at a low oversampling rate, so as to obtain the radiation characteristics of AUT with high accuracy. In particular, even if the quantity of 


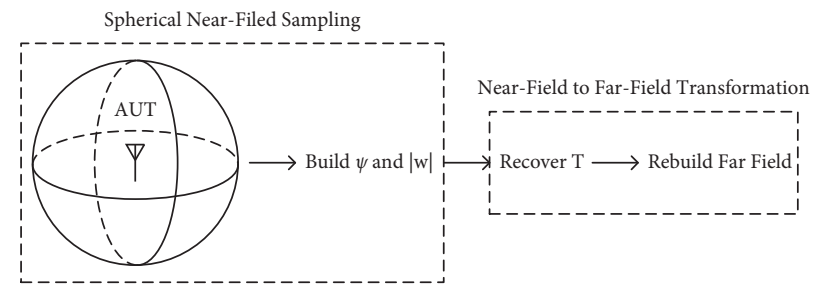

Figure 1: Spherical near-field measurement steps.

nonzero elements in SMC is uncertain, the proposed algorithm is still efficient in solving the problem without a priori knowledge of the sparsity level of the signal to be estimated.

For the algorithm to possess the abovementioned ability of recovering the complex vector under the condition of unknown sparsity level, the following strategies are adopted. First, the sparsity of the signal is guaranteed by $\ell_{1}$-norm. Unlike the ordinary definition of $\ell_{1}$-norm for complex vectors, we choose to decrease the dimension of the complex model to a real number by separating the real and imaginary parts of the signal to reduce the sparsity level. Afterward, a composite vector is rebuilt and its $\ell_{1}$-norm is inserted into the objective function as a sparse constraint. As for the problem with unknown sparsity level, the sparse regularization parameter is set to decrease from a larger value, allowing the whole optimization process to be decomposed into multiple suboptimization problems similar to LASSO. In the early stage of iteration, the sparsity of the solution is fulfilled first. With the downward adjustment of the weight of the sparse regularization term, the error between the recovered and the measured amplitude is constantly diminished.

Meanwhile, in order to give full play to the performance advantages of the proposed algorithm, it is essential to determine an effective near-field sampling method. As illustrated in Figure 1, different sampling strategies will construct different measurement matrices and amplitude vectors, which will indirectly affect the recovery accuracy of the algorithm. Since the utilization of the measurement matrix with lower correlation can deliver more information, the success rate of the algorithm in recovering SMC is raised. Therefore, we determine an optimal spherical near-field sampling method that matches the algorithm by calculating the correlation of the measurement matrix and comparing the accuracy of the obtained AUT's radiation pattern. The parameters to be specified include the number of spheres, the distribution of the sampling points, radius difference, and the polarization mode.

\section{The Improved Phase Recovery Algorithm}

\subsection{Algorithm Introduction}

3.1.1. Overall Framework of the Algorithm. The sparse phase recovery problem [13] can usually be described as

$$
\begin{aligned}
& \min \|x\|_{1}, \\
& \text { subject to } b_{i}=\left|a_{i}^{*} x\right|, \quad 1 \leq i \leq m .
\end{aligned}
$$

Inspired by the penalty function method to solve basis pursuit, identical to [21], we apply penalty functions acting on the equation constraints in problem (2), which transforms the phase recovery of the sparse signals into solving multiple optimization problems similar to LASSO:

$$
\min f:=\lambda\|x\|_{1}+\||A x|-b\|_{2}^{2} .
$$

Regarding the value of the regularization parameter $\lambda$, we set it to a value that is decreased from a larger one, i.e., $\lambda_{k}=\gamma \lambda_{k-1}, \gamma \in(0,1)$ as a reduction factor.

The advantage is that at the beginning of the iteration, the algorithm first optimizes $\|x\|_{1}$, thus ensuring the sparsity of the solution due to the larger value of $\lambda$. As the iteration proceeds, $\lambda$ monotonically decreases, enabling the algorithm to pay more attention to reducing the error $\||A x|-b\|_{2}^{2}$.

In conclusion, the general framework of the proposed algorithm can be described as follows:

3.1.2. Modification of the Objective Function. As with amplitude flow, we still use the gradient descent method to solve suboptimization problems. Meanwhile, in order to make the algorithm applicable to recover complex sparse signals, we have to modify the objective function in equation (3).

(1) Sparsity Constraint. We emphasize that the purpose of adding sparse constraints is not to use the measured data far less than the signal length for phase recovery but to enable the algorithm to seek the sparsest solution from the feasible region at a low oversampling rate.

Since the real and imaginary parts are the two orthogonal components of the complex signal, they usually behave as either simultaneously 0 or not 0 [22]. Certainly, SMC also satisfies the abovementioned properties. Taking a dipole array with reflector plane as an example, we plot the magnitude distribution of real and imaginary parts of $T_{1}$ and $T_{2}$ as shown in Figure 2, which confirms the conclusion. Therefore, we separate the real and imaginary parts of the signal to be evaluated to lower its sparsity level and enhance the recovery capability, which is the primary reason why we reduce the dimension of the complex system to real numbers.

To facilitate the representation of the problem, we defined $(x)_{\mathbb{R}} \in \mathbb{R}^{n}$ as the real part of the complex sparse signal $x,(x)_{\rrbracket} \in \mathbb{R}^{n}$ corresponding to its imaginary part; hence,

$$
x=(x)_{\mathbb{R}}+i(x)_{\mathbb{1}} .
$$


(1) Initial estimation $x^{0}$, final value $\lambda$, initial value $\lambda_{0}$, reduction factor $\gamma \in(0,1), k \longleftarrow 0$

(2) while $\lambda_{k} \geq \lambda$ do

(3) with $x^{k}$ as the initial value, solve the problem: $\min f:=\lambda_{k}\|x\|_{1}+\||A x|-b\|_{2}^{2}$.

(4) if $\lambda_{k}=\lambda$ then

(5) stop iteration, output $x^{k+1}$

(6) else

(7) update the penalty factor: $\lambda_{k+1}=\max \left\{\lambda, \gamma \lambda_{k}\right\}$.

(8) $k \longleftarrow k+1$

(9) end if

(10) end while

Algorithm 1: The general framework of the proposed algorithm.
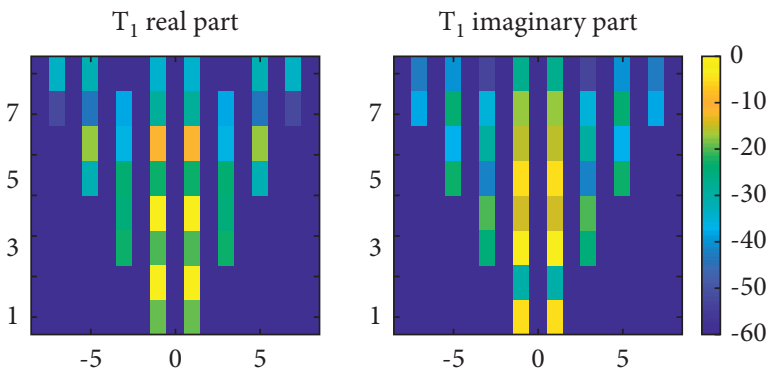

(a)

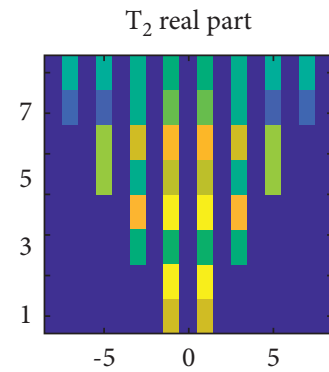

(b)

b)

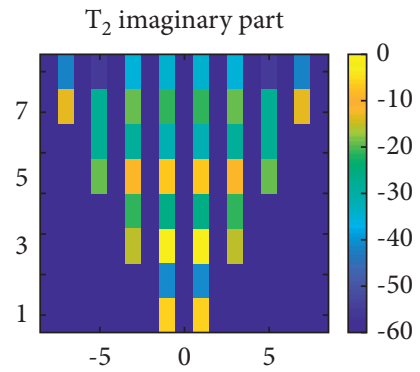

Figure 2: The magnitude distribution of real and imaginary parts of (a) $T_{1}$ and (b) $T_{2}$.

$\widetilde{x} \triangleq\left[(x)_{\mathbb{R}}^{T}(x)_{\square}^{T}\right]^{T} \in \mathbb{R}^{2 n}$ denotes the composite vector of the real and imaginary parts of $x$.

The sparsity constraint is defined as $\ell_{1}$-norm of the composite vector $\tilde{x}$ :

$$
\|\tilde{x}\|_{1}=\sum_{i=1}^{2 n}\left|\tilde{x}_{i}\right|
$$

Since $\|\tilde{x}\|_{1}$ cannot derive its gradient at $\tilde{x}_{i}=0$, it is impossible to directly solve the original problem using the gradient method. Therefore, it is considered to be approximated by the smooth function [23] of the following equation

$$
\ell_{\delta}\left(\tilde{x}_{i}\right)= \begin{cases}\frac{1}{2 \delta} \widetilde{x}_{i}^{2}, & \left|\widetilde{x}_{i}\right|<\delta \\ \left|\tilde{x}_{i}\right|-\frac{\delta}{2}, & \text { otherwise. }\end{cases}
$$

We adopt $\mathscr{L}_{\delta}(\tilde{x})=\sum_{i=1}^{2 n} \ell_{\delta}\left(\tilde{x}_{i}\right)$ to replace $\|\tilde{x}\|_{1}$ as the sparse constraint in the objective function.

(2) Loss Function. The loss function is still specified as the square of the $\ell_{2}$-norm of the difference between the recovery and the measured amplitude.

$$
L(x)=\sum_{i=1}^{m}\left(\left|a_{i}^{*} x\right|-b_{i}\right)^{2} .
$$

3.1.3. The Solution Process of the Suboptimization Problem. Each suboptimization problem is iteratively solved by the gradient descent method similar to equation (8). The complex vector $\tilde{x}$ is continuously updated until the objective function is minimized.

$$
\tilde{x}_{t+1}=\tilde{x}_{t}-\mu_{t} \nabla_{\widetilde{x}} f,
$$

where $\mu_{t}$ is the appropriate step-size adjusted with the number of iterations [24].

The first half elements of the resulting composite vector $\tilde{x}$ are the real part of $x$, and the second half corresponds to its imaginary part; thus,

$$
x=\left.\widetilde{x}\right|_{1: n}+\left.i \widetilde{x}\right|_{n+1: 2 n} .
$$

(1) Initial Estimation. In essence, the algorithm is still dedicated to solving a nonconvex optimization problem. To ensure that the final solution converges to the global minimum, an initial vector close to the real solution needs to be acquired by means of the eigenvalue method [25].

In this study, the optimized spectral initializer is applied [26]. First, the measured amplitude is preprocessed according to the following equation.

$$
\mathscr{T}_{\rho}\left(b_{i}\right)=\frac{b_{i}-1}{b_{i}+\sqrt{\rho}-1},
$$

where $\rho=m / n$.

Second, the constant $\beta$ associated with the measurement matrix and the sampling amplitude is calculated. 


$$
\beta^{2}=n \frac{\sum_{i} b_{i}}{\sum_{i}\left\|a_{i}\right\|^{2}}
$$

Let $x_{0}$ be the eigenvector corresponding to the maximum eigenvalue of the given matrix:

$$
X=\frac{1}{m} \sum_{i=1}^{m} \mathscr{T}_{\rho}\left(b_{i}\right) a_{i} a_{i}^{*} .
$$

Then $\left\|x_{0}\right\|=\beta$ is set to scale the estimation vector so that its $\ell_{2}$-norm is roughly consistent with the true solution.

The real and imaginary parts of $x_{0}$ are extracted, thus reconstructing the composite vector

$$
\tilde{x}_{0} \triangleq\left[\left(x_{0}\right)_{\mathbb{R}}^{T}\left(x_{0}\right)_{\mathbb{1}}^{T}\right]^{T} .
$$

(2) Gradient of the Objective Function. The gradient calculation of the objective function will be divided into two parts: the sparse constraint $\mathscr{L}_{\delta}(\tilde{x})$ and the loss function $L(x)$.
In particular, we will separately compute the gradient of $L(x)$ to the real and imaginary part of $x$. Thus, a composite vector of gradients is defined to accomplish the update of $\tilde{x}$.

First, the gradient of the smoothed $\ell_{1}$-norm to the composite vector $\tilde{x}$ is given by

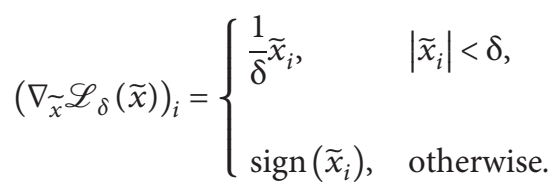

As for the loss function, to facilitate the description of its gradient calculation procedure, $(A)_{\mathbb{R}} \in \mathbb{R}^{m \times n}$ represents the real part of the complex measurement matrix and $(A)_{\square} \in \mathbb{R}^{m \times n}$ corresponds to its imaginary part. In addition, $\hat{a}_{i}$ denotes the transpose of i-th row of $(A)_{\mathbb{R}}$ and the transpose of the $\mathrm{i}$-th row of $(A)_{\mathbb{1}}$ is defined as $\widehat{a}_{i}$, $i=1,2 \ldots m$. Therefore,

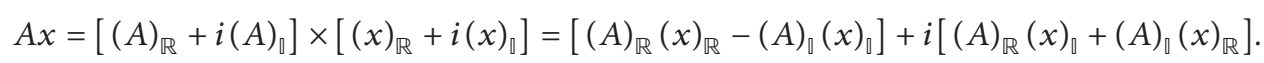

Then, its real and imaginary parts are defined as follows:

$$
\overline{A x}_{i}=|A x|_{i}=\sqrt{|\widehat{A x}|^{2}+|\widehat{A x}|^{2}}, \quad i=1,2 \ldots m .
$$

$$
\begin{aligned}
\widehat{A x} & =(A)_{\mathbb{R}}(x)_{\mathbb{R}}-(A)_{\mathbb{}}(x)_{\mathbb{\square}}, \\
\widehat{A x} & =(A)_{\mathbb{R}}(x)_{\mathbb{\square}}+(A)_{\mathbb{}}(x)_{\mathbb{R}} .
\end{aligned}
$$

As previously stated, the gradients of the loss function to $(x)_{\mathbb{R}}$ and $(x)_{\mathbb{Q}}$ are calculated, respectively. The corresponding results are as follows: follows:

Therefore, the recovered amplitude can be expressed as

$$
\begin{aligned}
& \nabla_{(x)_{\mathbb{R}}} L(x):=\sum_{i=1}^{m} \frac{2 \cdot\left(\left|a_{i}^{*} x\right|-b_{i}\right)}{\overline{A x}_{i}} \cdot\left(\widehat{A} x_{i} \cdot \widehat{a}_{i}+\widehat{A x}_{i} \cdot \widehat{a}_{i}\right), \\
& \nabla_{(x)_{0}} L(x):=\sum_{i=1}^{m} \frac{2 \cdot\left(\left|a_{i}^{*} x\right|-b_{i}\right)}{\overline{A x}_{i}} \cdot\left(-\widehat{A} x_{i} \cdot \widehat{a}_{i}+\widehat{A x}_{i} \cdot \widehat{a}_{i}\right) .
\end{aligned}
$$

if $\overline{A x}_{i}=0$.

The convention $\nabla_{(x)_{\mathbb{R}}} L(x)$ and $\nabla_{(x)_{\|}} L(x)=0$ is adopted,

Thus, the composite vector of the gradient is defined as follows:

$$
\nabla_{\tilde{x}} L(\tilde{x})=\left[\left(\nabla_{(x)_{\mathbb{R}}} L(x)\right)^{T}\left(\nabla_{(x)_{\mathbb{1}}} L(x)\right)^{T}\right]^{T} \in \mathbb{R}^{2 n}
$$

In summary, the gradient of the objective function of each suboptimization problem is as follows:

$$
\nabla_{\tilde{x}} f(\tilde{x})=\lambda \nabla_{\widetilde{x}} \mathscr{L}_{\delta}(\tilde{x})+\underset{\mathrm{x}}{\nabla \sim L}(\tilde{x}) .
$$

(3) Determination of the Iteration Step-Size. To improve the convergence properties of the gradient descent method in equation (8), we adopt the Barzilai-Borwei step [16], which permits adaptive adjustment of the step size only based on the information of the two adjacent iteration points and their gradients.

First,

$$
\begin{aligned}
& s^{\tau-1} \triangleq \tilde{x}^{\tau}-\tilde{x}^{\tau-1} \\
& y^{\tau-1} \triangleq \nabla_{\tilde{x}} f\left(\tilde{x}^{\tau}\right)-\nabla_{\tilde{x}} f\left(\tilde{x}^{\tau-1}\right) .
\end{aligned}
$$


The step size of the Barzilai-Borwein form is expressed as follows:

$$
\begin{aligned}
& \mu_{\mathrm{BB} 1}^{\tau} \triangleq \frac{\left(s^{\tau-1}\right)^{T} y^{\tau-1}}{\left(y^{\tau-1}\right)^{T} y^{\tau-1}} \\
& \mu_{\mathrm{BB} 2}^{\tau} \triangleq \frac{\left(s^{\tau-1}\right)^{T} s^{\tau-1}}{\left(s^{\tau-1}\right)^{T} y^{\tau-1}} .
\end{aligned}
$$

Therefore, the following two iteration formats for the Barzilai-Borwein method are obtained as follows:

$$
\begin{aligned}
& \tilde{x}_{t+1}=\tilde{x}_{t}-\mu_{\mathrm{BB} 1}^{\tau} \nabla_{\tilde{x}} f(\tilde{x}), \\
& \tilde{x}_{t+1}=\tilde{x}_{t}-\mu_{\mathrm{BB} 2}^{\tau} \nabla_{\tilde{x}} f(\tilde{x}) .
\end{aligned}
$$

Overall, the solution steps for each suboptimization problem can be summarized as follows.

3.2. Numerical Results for Gaussian Data. Before the algorithm is applied to the spherical NFFFT problem, numerical tests are carried out on Gaussian data to demonstrate the performance of the improved algorithm. The measurement matrix is randomly generated. The real and imaginary parts of the observation vector and the vector to be estimated are independently subject to Gaussian distribution.

3.2.1. Test 1. Fixing the number of nonzero elements in the sparse vector $x \in \mathbb{C}^{n}$ as $p$, the recovery performance of the algorithm is investigated when $m$ measurements are taken.

Since SMC includes more nonzero elements, the sparsity level $s=p / n$ is relatively higher. As corroborated in [27], less than $15 \%$ of the spherical modes can contain $99.90 \%$ of the antenna's radiated power. Therefore, we set the sparsity level $s=p / n=10 \%$ and the length of the unknown vector $n$ to 100 .

The relative error is defined as follows:

$$
\text { Relative error }=\frac{\text { distance }\left(x_{\text {True }}, x_{\text {recovered }}\right)}{\left\|x_{\text {True }}\right\|} .
$$

Then, $(m / n)$ increases from 0.1 to 3.5 in the step of 0.1 , and 100 independent trials are carried out, respectively. Success is claimed for a trial if the returned estimate incurs a relative error less than $10^{-5}$ [24]. The numerical results depicted in Figure 3 reveal that the proposed algorithm achieves an approximate $100 \%$ success rate when the sparsity level is 0.1 and the oversampling rate $(m / n) \geq 2.3$.

Furthermore, numerical trials are conducted using PhasePack [28] for amplitude flow, truncated amplitude flow, reweighted amplitude flow [29], Wirtinger flow, reweighted Wirtinger flow [30], Fienup [31], and G-S under the same conditions. The related results are also presented in Figure 3.

3.2.2. Test 2. This experiment mainly focuses on the capability of the proposed algorithm to recover complex signals with various sparsity levels for a fixed $m$. Figure 4 depicts the numerical results, indicating that the proposed algorithm is able to guarantee the accurate recovery of complex vectors at a sparsity level less than 0.09 . As the number of nonzero elements of the signal grows, the success rate of the algorithm gradually decreases, and it can support the recovery of complex vectors at a sparsity level of 0.36 at most.

In conclusion, when Gaussian data are taken for testing, compared with ordinary phase recovery algorithms, the proposed algorithm can still deliver accurate estimates at low oversampling rates. Besides, compared with sparse phase recovery algorithms such as CoPRAM, SPARTA, and CPRL, the proposed algorithm behaves well for complex sparse vectors containing more nonzero elements.

3.3. Determination of the Spherical Near-Field Sampling Strategy. Since the near-field sampling strategy will directly dictate the measurement matrix and amplitude vector, which in turn affect the performance of the algorithm, before exhibiting the performance advantage of the algorithm in recovering SMC, it is essential to determine an effective near-field sampling method.

We take a $4 \times 2$ dipole antenna array with a reflector as AUT. The total amount of spherical modes $n$ is 160 . In the below analysis of the various near-field sampling methods, an oversampling rate of 2.5 is kept invariant.

The parameters involved in the spherical near-field sampling process include the number of spheres, the distribution of measurement points, the radius difference, and the polarization method, which will be individually investigated afterward. Similar to [27], in order to evaluate each sampling scheme, except for comparing the accuracy of the extrapolated far field, the correlation of the measurement matrix is additionally considered as an assessment index. In short, we perceive that employing a measurement matrix with high correlation will contribute to poor recovery performance.

Defining the row vector of the measurement matrix $\psi$ as $\psi_{i}$, and $i=1,2 \ldots m$, the correlation coefficient can be computed by

$$
\mu_{i, j}=\frac{\left|\left\langle\psi_{i}, \psi_{j}\right\rangle\right|}{\left\|\psi_{i}\right\|_{2}\left\|\psi_{j}\right\|_{2}}, \quad i \neq j
$$

3.3.1. The Number of Sampling Spheres. Until now, there have been no strictly practical guidelines on how to acquire independent near-field sampling data to meet the requirements. Intuitively, oversampling just on a single sphere is likely to add linearly dependent rows to the measurement matrix, resulting in a marginal improvement in the recovery of SMC. Hence, we decide to sample half of the data on the other concentric sphere to compensate for the lack of information inherent in phaseless measurements. We also exploit the correlation to illustrate the validity of the twosphere sampling strategy. The corresponding two vectors with $\mu$ greater than 0.4 are claimed to be strongly correlated.

The correlation of the measurement matrices derived from the single and double spherical sampling methods is 


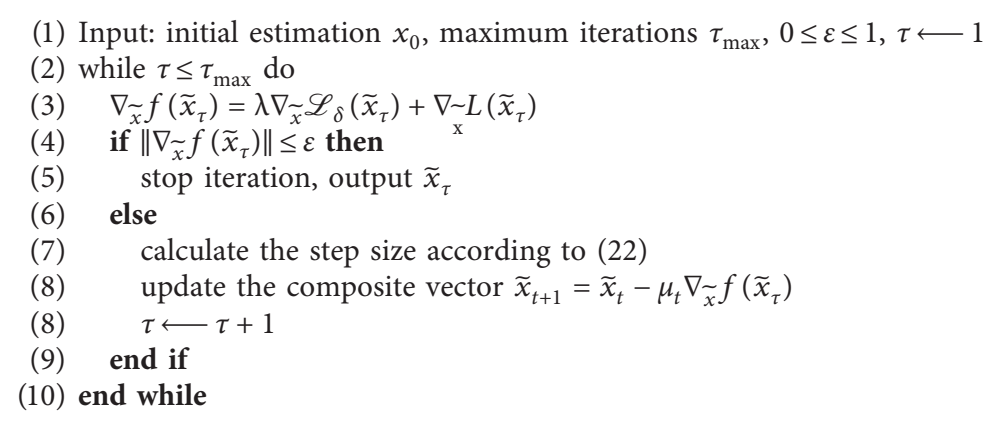

Algorithm 2: The process of solving the suboptimization problem.

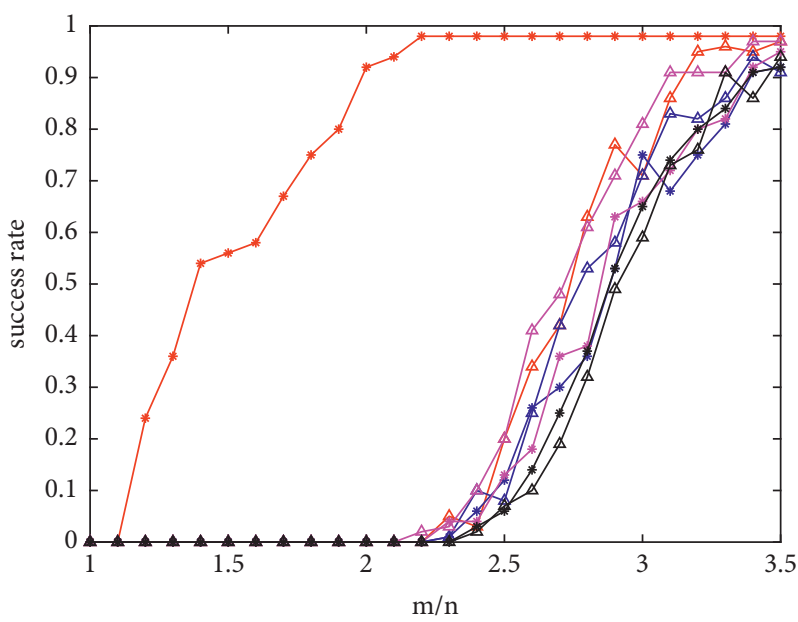

$$
\begin{array}{lc}
\rightarrow-\text { New Algorithm } & * \text { - Wirtinger Flow } \\
\triangle \text { Reweighted Amplitude } & \triangle \text { Reweighted Wirtinger } \\
& \text { Flow } \\
\rightarrow \text { Truncated Amplitude } & \rightarrow \text { Fienup } \\
\text { Flow } & \triangle \text { G-S } \\
\triangle \text { Amplitude Flow } &
\end{array}
$$

FIgURE 3: Success rates for algorithms at a fixed sparsity level and the length of an unknown vector.

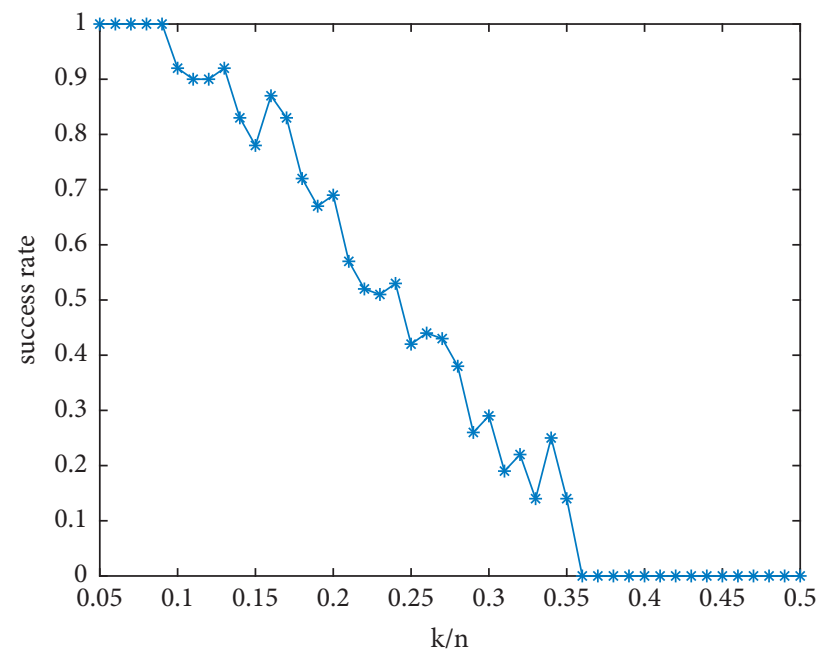

FIGURE 4: Success rates for the proposed algorithm with fixed $m$ and the length of the unknown vector. 
reported in Table 1. It is clear that the two-sphere strategy can significantly diminish the correlation to provide more additional information. The radiation pattern computed by the proposed algorithm depicted in Figure 5 also demonstrates the above analysis.

3.3.2. The Distribution of Sampling Points. As for the distribution of sampling points on each sphere [32-34], we mainly investigate the three forms of spiral, Fibonacci, and Hammersley as illustrated in Figure 6.

Each sphere can adopt one of the three forms, and thus, two spheres incur nine sampling methods listed in Table 2. The corresponding near field is processed utilizing the proposed algorithm, and the derived radiation pattern is given in Figure 7. Considering the correlations of the measurement matrix comprehensively summarized in $\mathrm{Ta}-$ ble 2, it can be noticed that when both the two spheres are in the form of spiral or Fibonacci distributions, the obtained measurement matrix exhibits lower correlations, thereby yielding the extrapolated far field with higher accuracy.

Particularly, compared with Fibonacci, when the sampling points are distributed as a spiral, the measurement trajectory of the positioning device achieves a lower complexity, leading to a shortened measurement time. In general, the spiral form is more applicable for spherical nearfield sampling.

3.3.3. The Radius Difference. The radius difference between the two spheres is also a key factor impacting the transformation accuracy, which is set to $1.5,2,2.5$, and 3 times the wavelength, respectively, in this study. The results shown in Table 3 demonstrate that the correlation between the row vectors of the measurement matrix is extremely high due to the smaller radius difference. With the separation of the two spheres, the independence between the two scans is gradually enhanced, enabling the electric field amplitude to experience sufficient variation and the extrapolation of the far field to become more precise, which can be observed in Figure 8 .

3.3.4. Polarization Mode. So far, we have only considered the location of the sampling points, whereas the polarization of the probe is likewise an additional parameter to be determined in near-field measurements. Three major possibilities will be considered for further analysis:

(1) One fixed polarization for all sampling points $(\chi=0$ or $\chi=\pi / 2)$.

(2) Two orthogonal polarizations at each sampling point $(\chi=0$ and $\chi=\pi / 2)$.

(3) Randomly chosen fixed polarization at each sampling point $(\chi=0$ or $\chi=\pi / 2)$.

For case 1, as expected, measurements with only one fixed polarization cannot afford correct solutions to SMC. The polarization mode introduced in case 2 is adopted in the above research on different sampling strategies. The results reveal that it can support stable near-field to far-field transformations. As for case 3, the total quantity of sampled data is maintained invariant and the sampling points are distributed in the form of a spiral. As illustrated in Figure 9, a fixed polarization is randomly chosen at each sampling point. Besides, the measured $E_{\theta}$ and $E_{\varphi}$ are guaranteed to be of the same amount.

Twenty random trials are conducted based on the above settings. Figure 10 only presents the calculated radiation pattern for 10 of them, which reveals that polarization will have a slight impact on the transformation precision, but the accurate reconstruction of the main lope can be realized with high probability.

Based on the above analysis, an optimized near-field sampling method is concluded: the measurement points are distributed on two concentric spheres in the form of a spiral. Two orthogonal polarizations are acquired at each sampling point.

\section{Numerical Results for SMC}

The above sampling strategy is applied to acquiring the nearfield data. SMC is solved by the proposed algorithm and other phase recovery algorithms. The performance advantage of the proposed algorithm is demonstrated by comparing the accuracy of the extrapolated far field. The two radiation structures, namely, dipole array and horn antenna, are investigated.

First, AUT as a $3 \times 3$ dipole array with reflector is specified, and the total number of its spherical mode is 240 . The oversampling rate is set to 2.5 , and the difference between the two sphere to $6 \lambda$. In the beginning, GS, Wirtinger flow, reweighted Wirtinger flow, amplitude flow, truncated amplitude flow, reweighted amplitude flow, and PhaseLamp are utilized to derive SMC. The reconstructed far-field strengths $E_{\theta}$ and $E_{\varphi}$ are depicted in Figures 11 and 12, respectively.

In conjunction with the radiation pattern depicted in Figure 13, it can be concluded that the accuracy of the extrapolated far field is unsatisfactory at an oversampling rate of 2.5, if the ordinary phase recovery algorithm is applied.

The same near-field data are processed using the proposed algorithm. Figure 14 depicts the magnitude distribution of the recovered SMC, which indicates that the proposed algorithm is able to provide an accurate recovery, and thus, the solution is more consistent with the sparse properties. The reconstructed far-field intensity along with the radiation pattern is presented in Figures 15 and 16, respectively.

The maximum error of the extrapolated far field with a runtime of all the recovery algorithms is summarized in Table 4. All transformations have been performed on a single core of an Intel Core i7-6500K CPU running at $2.5 \mathrm{GHz}$. The results reveal that the algorithm proposed in this study can significantly improve the accuracy of the extrapolated far field.

Furthermore, similar to the proposed algorithm, the phaseless spherical near-field to far-field transformation algorithm in $[6,8,35]$ also requires the usage of the near field from two concentric spheres. By retaining the calculated phase information and replacing the measured amplitude of 
TABLE 1: The correlation of the measurement matrices derived from the single and double spherical sampling methods.

\begin{tabular}{lcc}
\hline Type & Amount of vector pairs with correlation greater than 0.4 & Amount of vector pairs with correlation greater than 0.3 \\
\hline Single sphere & 754 & 1000 \\
Two spheres & 218 & 242 \\
\hline
\end{tabular}

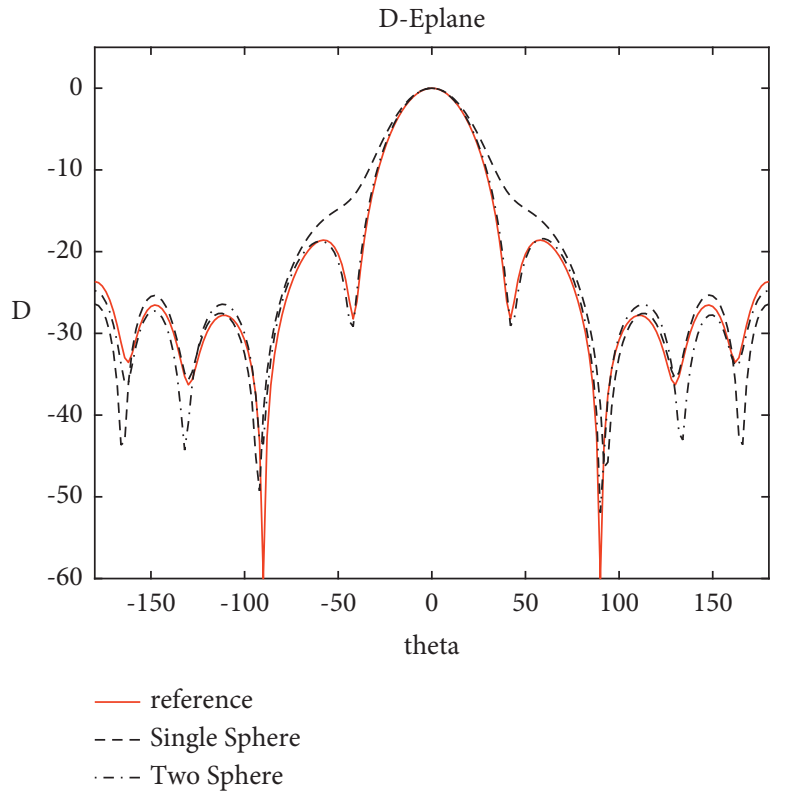

(a)

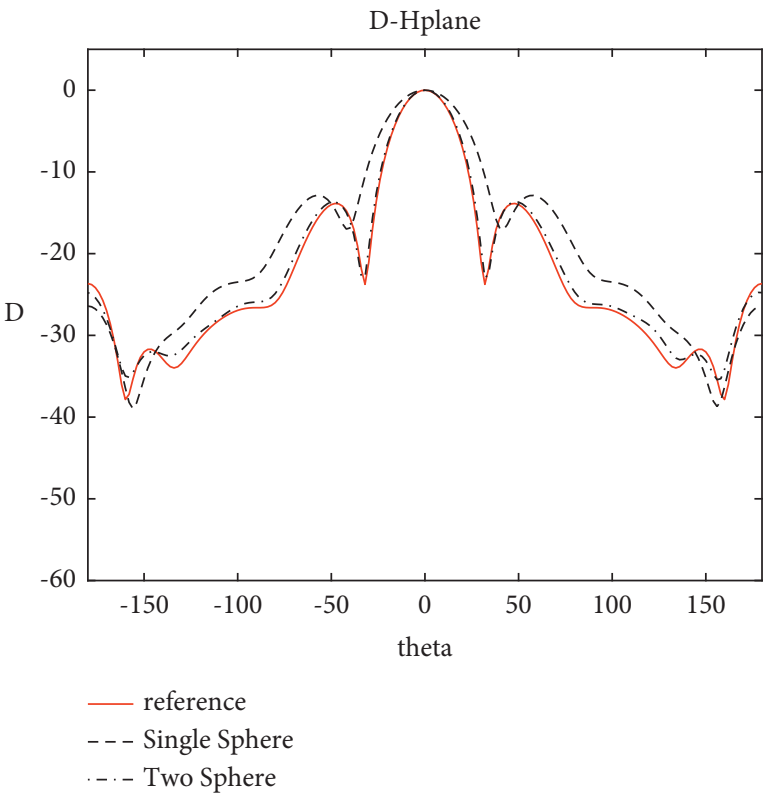

(b)

FIgURE 5: The radiation pattern corresponds to the single and double spherical sampling methods. The left and right figures represent the Eplane and H-plane, respectively. (a) E-plane. (b) H-plane.

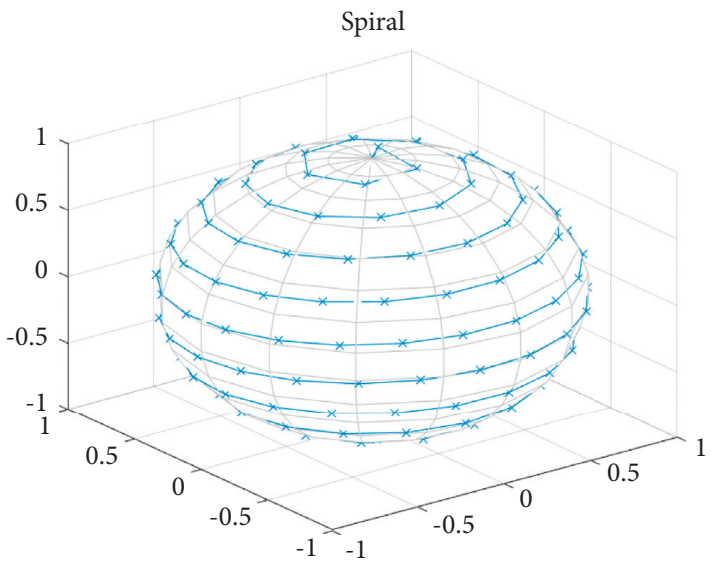

(a)

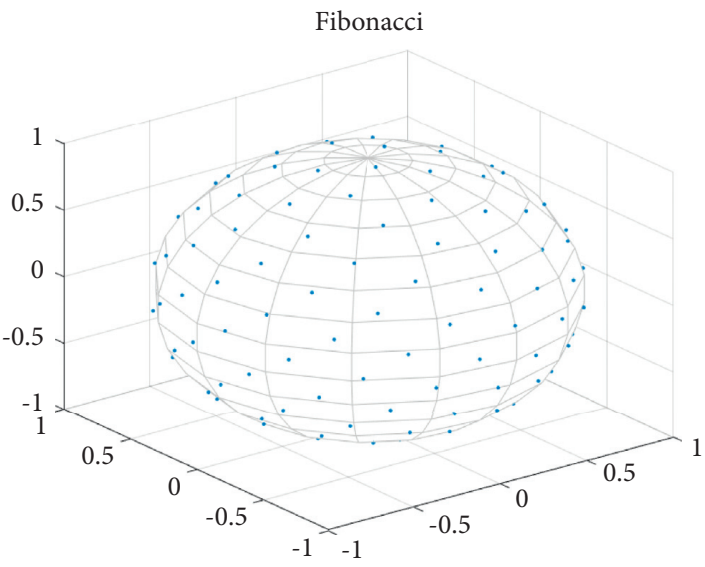

(b)

Figure 6: Continued. 


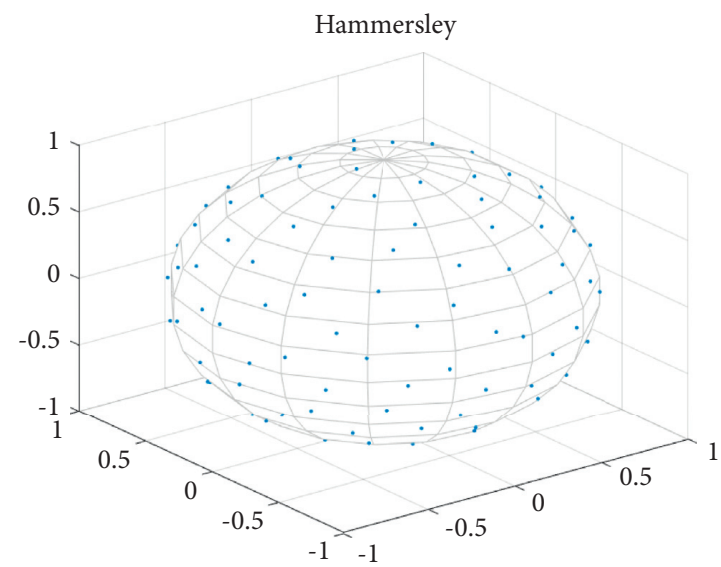

(c)

FIgURE 6: Schematic diagram of the three distribution forms of spherical sampling points. (a) Spiral. (b) Fibonacci. (c) Hammersley.

TABLE 2: Correlation of the measurement matrixes corresponding to the nine sampling methods.

\begin{tabular}{lcc}
\hline \multirow{2}{*}{ Type } & Amount of vector pairs with correlation greater than & Amount of vector pairs with correlation greater than \\
\hline spiral + spiral & 0.4 & 0.3 \\
fibonacci + fibonacci & 218 & 242 \\
hammersley + hammersley & 200 & 200 \\
spiral + fibonacci & 481 & 589 \\
spiral + hammersley & 384 & 479 \\
fibonacci + spiral & 437 & 608 \\
fibonacci + hammersley & 382 & 479 \\
hammesley + spiral & 422 & 549 \\
hammersley + fibonacci & 434 & 611 \\
\hline
\end{tabular}
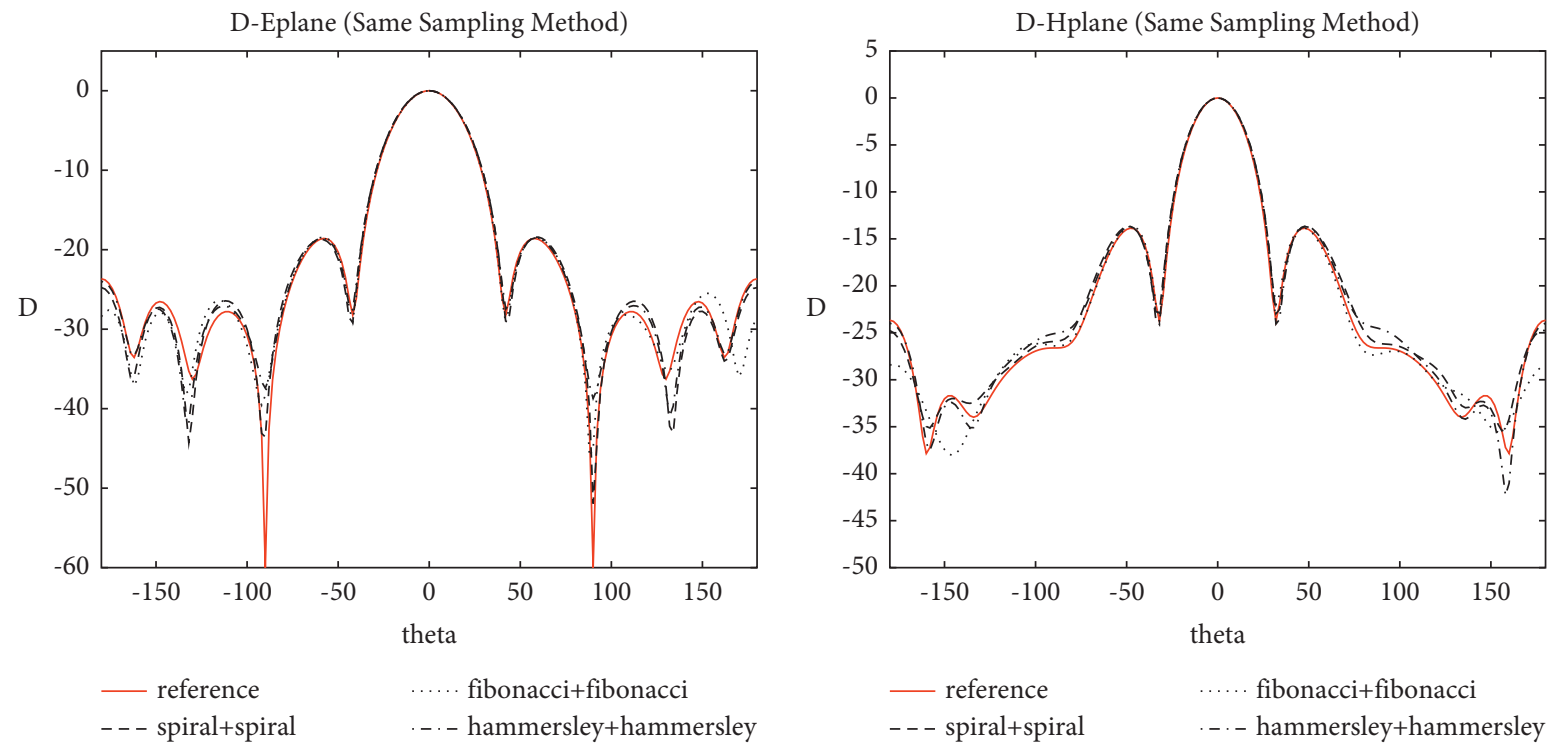

(a)

Figure 7: Continued. 

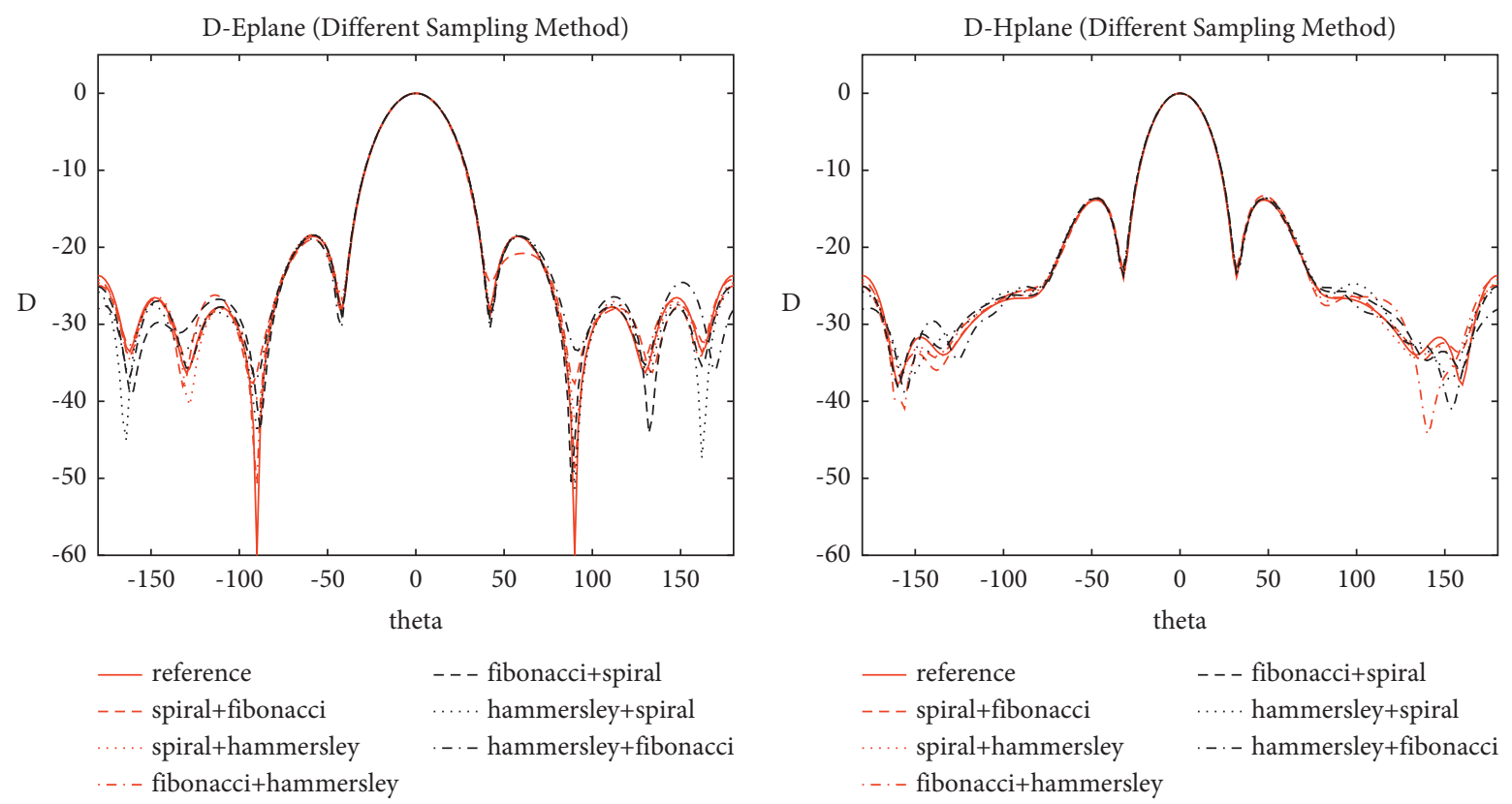

(b)

FIGURE 7: Radiation pattern of the dipole array corresponding to the nine sampling methods. (a) Radiation pattern corresponding to two spheres with the same distribution forms. The left and right sides of the figure represent E-plane and H-plane, respectively. (b) Radiation pattern corresponding to two spheres with different distribution form. The left and right sides of the figure represent E-plane and $\mathrm{H}$-plane, respectively.

TABLE 3: Correlation of the measurement matrix corresponding to different radius difference.

\begin{tabular}{lr}
\hline Radius difference/ $\lambda$ & Maximum correlation \\
\hline 1.5 & 0.9470 \\
2 & 0.9276 \\
2.5 & 0.9104 \\
3 & 0.8952 \\
\hline
\end{tabular}

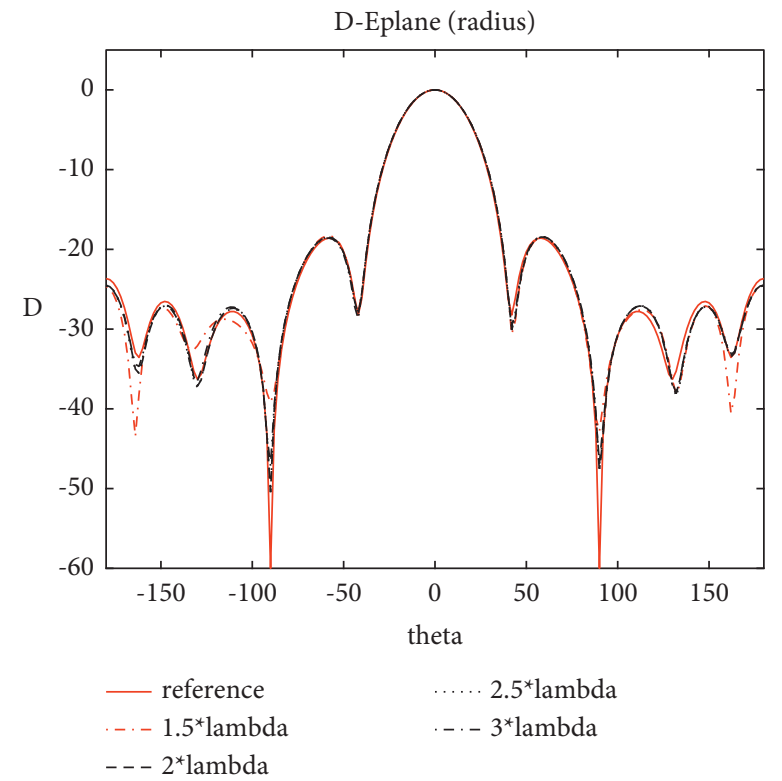

(a)

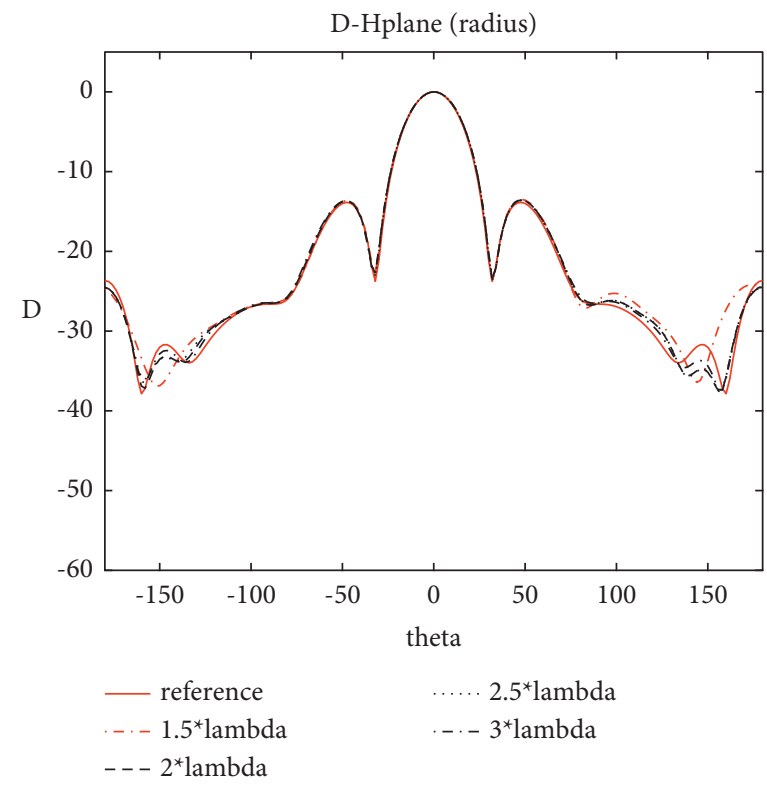

(b)

Figure 8: The radiation pattern corresponds to radius difference. (a) E-plane. (b) H-plane. 


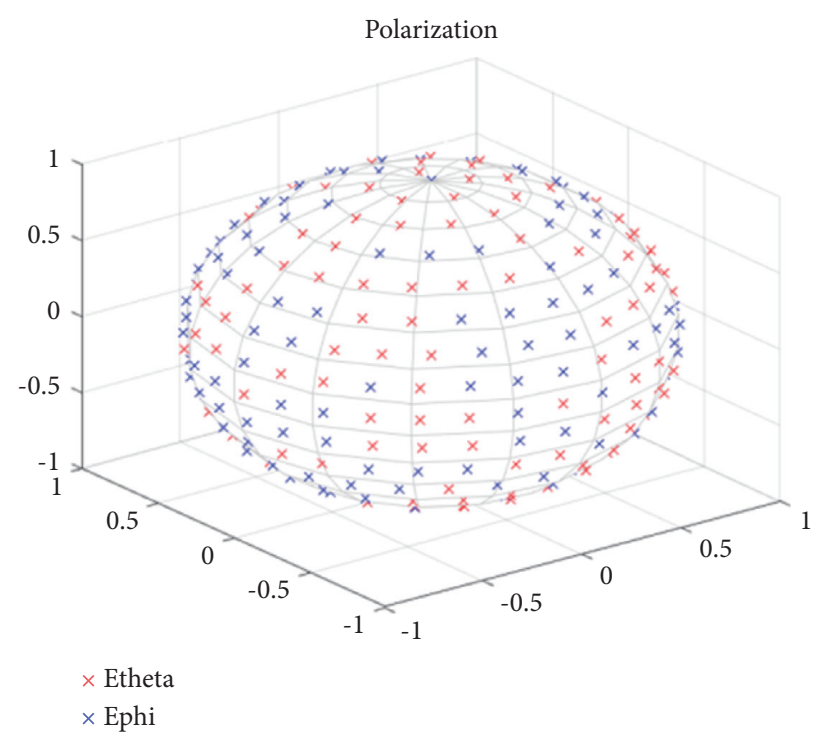

Figure 9: Sampling strategy described in case 3.

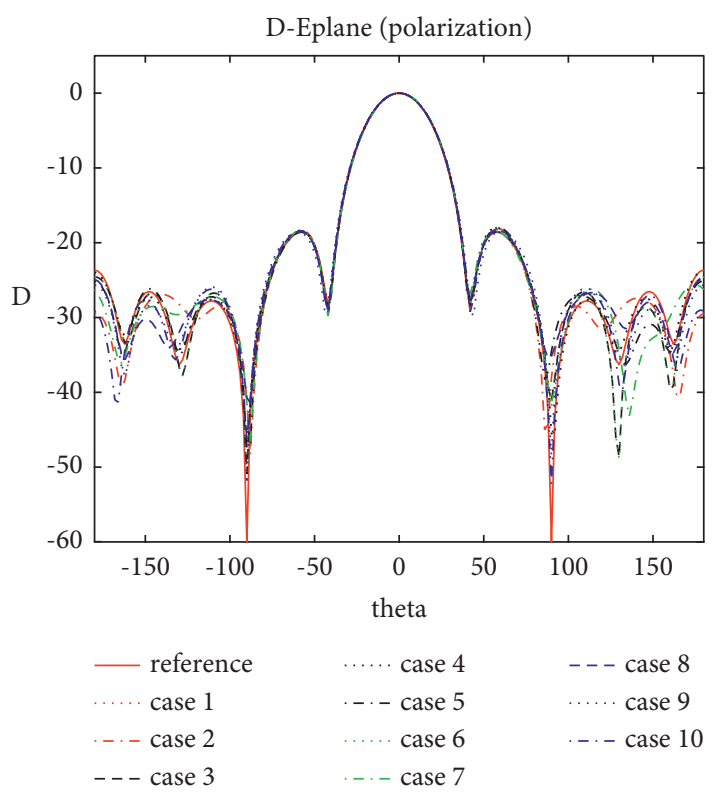

(a)

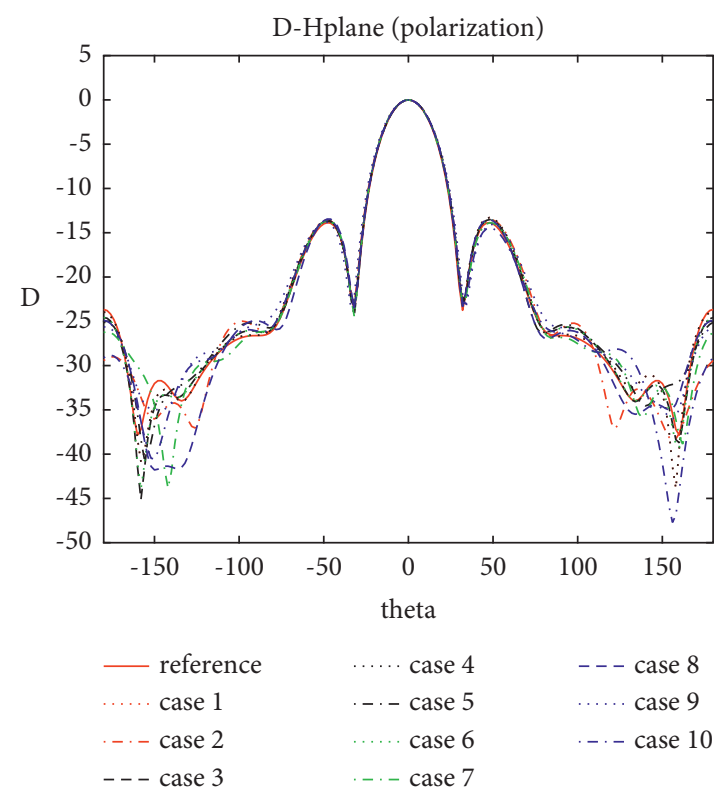

(b)

FIgURE 10: The radiation pattern corresponding to polarization mode described in case 3. (a) E-plane. (b) H-plane.

the electric field, the algorithm allows the two spheres to continuously exchange information and iteratively converge it, and finally, the SMC is solved.

However, the algorithm suffers from the problem of requiring a large quantity of measurement points and slow convergence. It also needs the near field to be strictly sampled in the equiangular form, resulting in the sampling point density low at the equator and high at the poles.

Therefore, the algorithm in this study turns to model the solution of SMC as a phase recovery problem, which avoids the tedious iterative process and can be well suited to the nonequiangular sampling methods.
For the $3 \times 3$ dipole array with reflector, Figure 17 depicts the extrapolated radiation pattern utilizing the algorithm in $[6,8,35]$. The proposed algorithm in this study performs better in terms of measurement, computation time, and farfield accuracy compared with it. The capabilities of the two algorithms are summarized in Table 5.

Moreover, a horn antenna is considered as AUT with a total number of spherical modes of 510. To guarantee that the algorithm can receive a high-quality initial estimate, the oversampling rate $m / n$ is set to 4 . The radius difference between the two measurement spheres corresponds to 5.483 $\lambda$. Compared with the extrapolated far-field results corresponding to other 

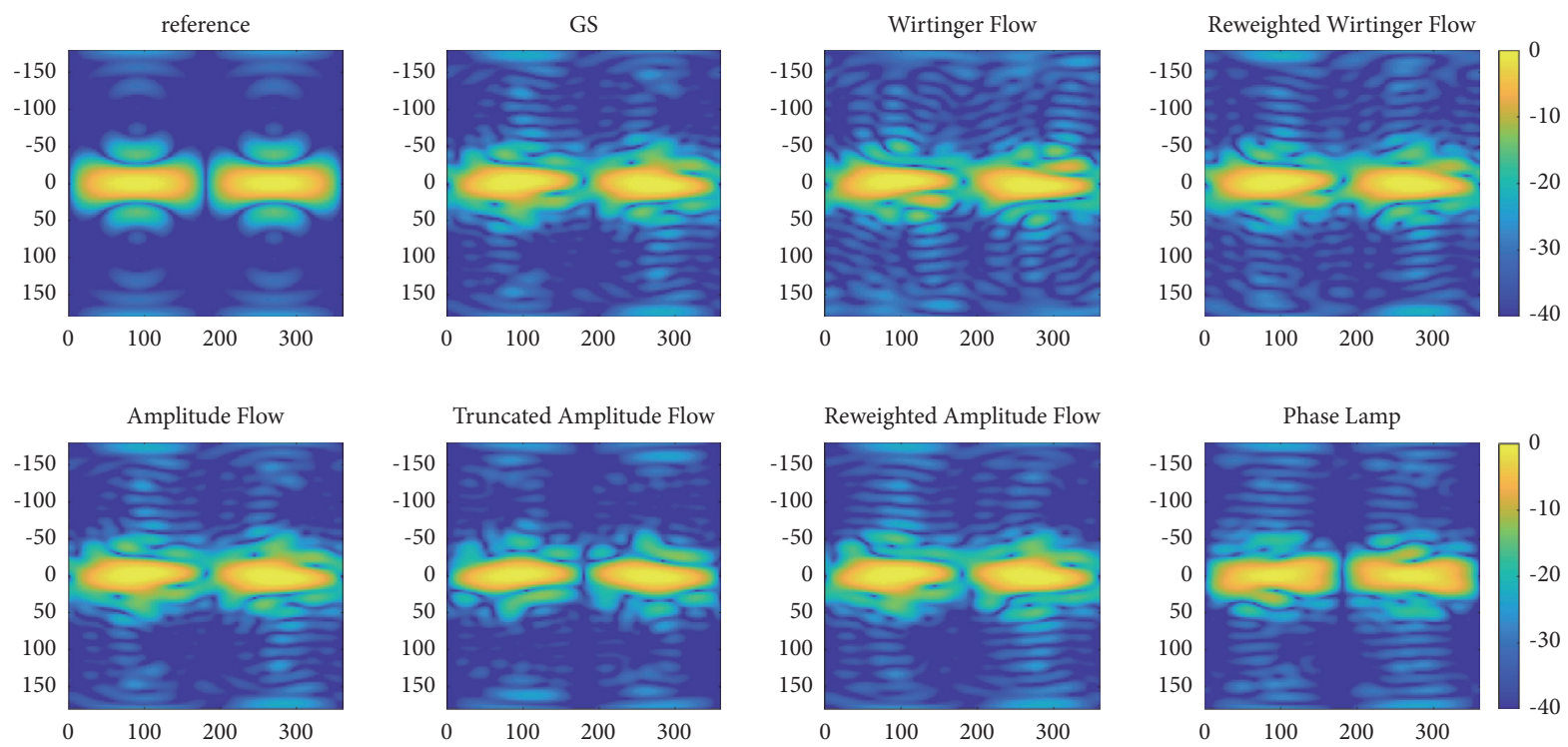

FigURE 11: The reconstructed $E_{\theta}$ of dipole array by adopting other phase recovery algorithms.
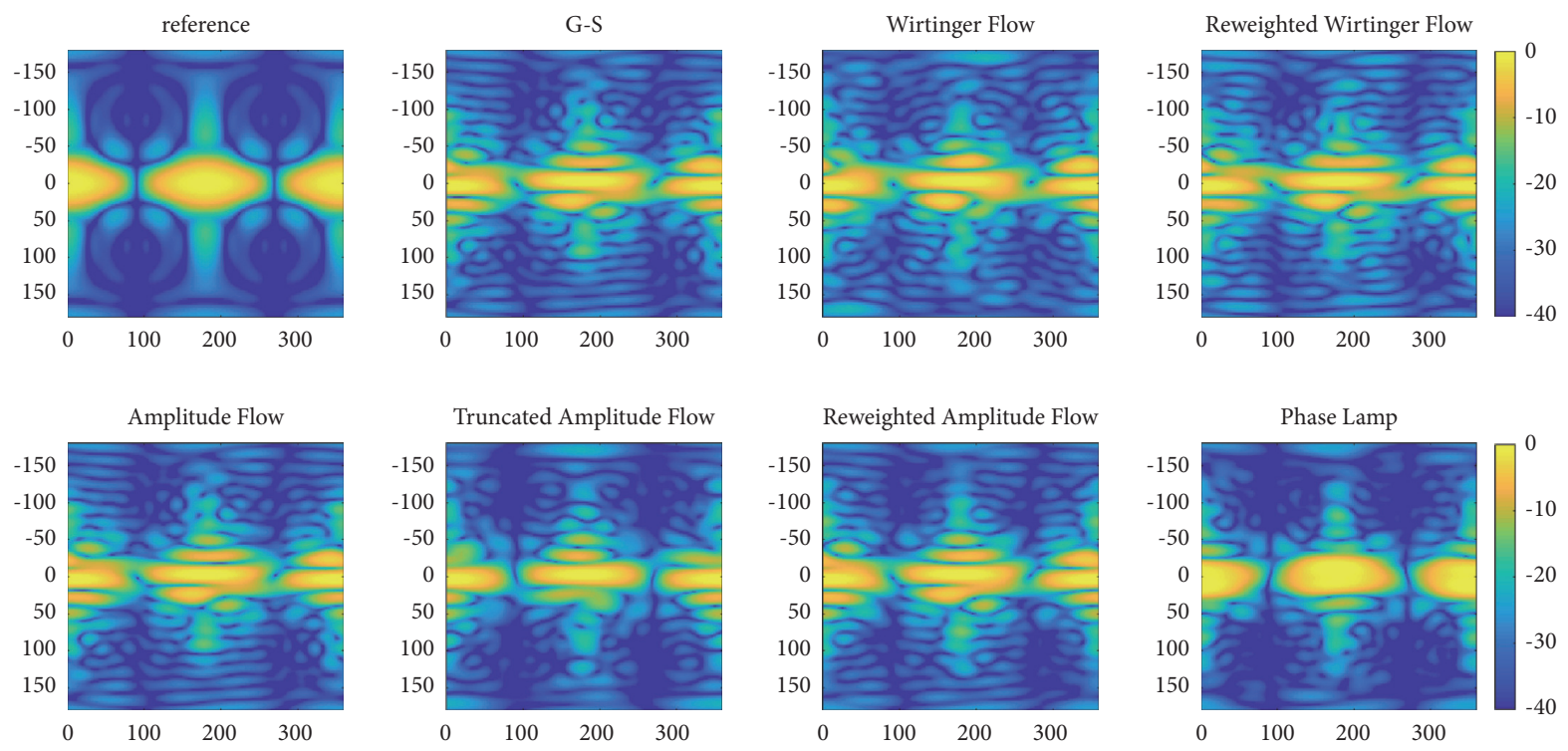

FIGURE 12: The reconstructed $E_{\varphi}$ of dipole array by adopting other phase recovery algorithms. 


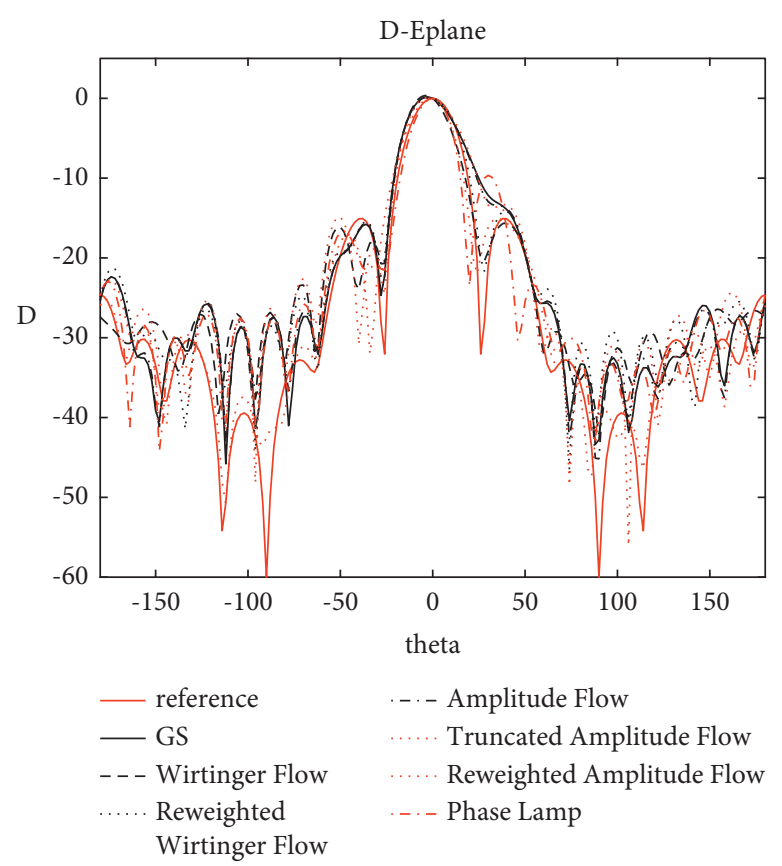

(a)

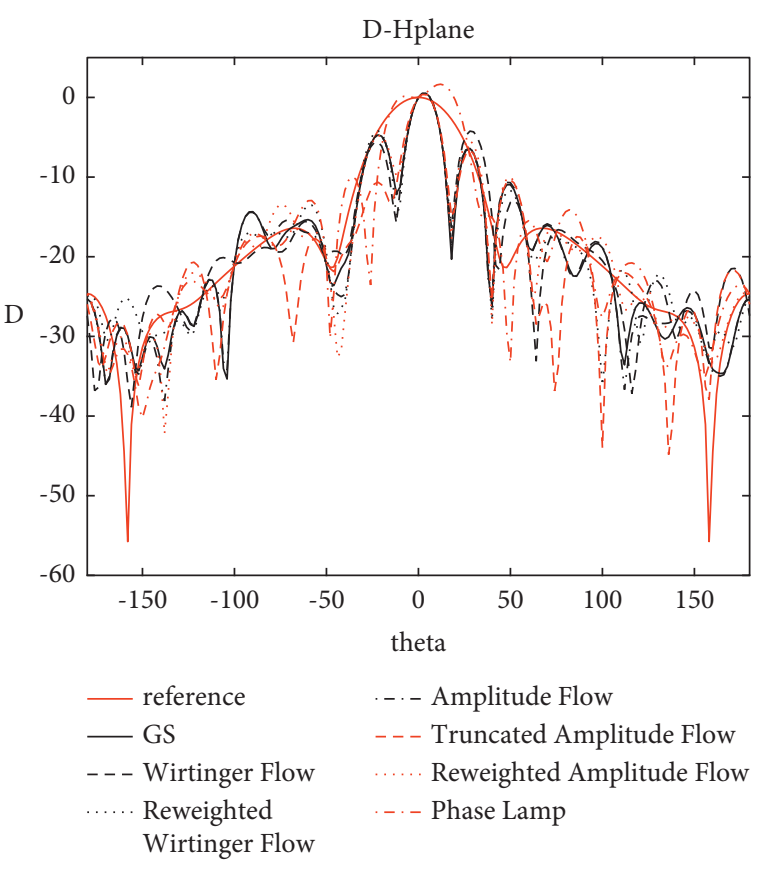

(b)

FIgURE 13: The obtained radiation pattern of dipole array by adopting other phase recovery algorithms. (a) E-plane. (b) H-plane.

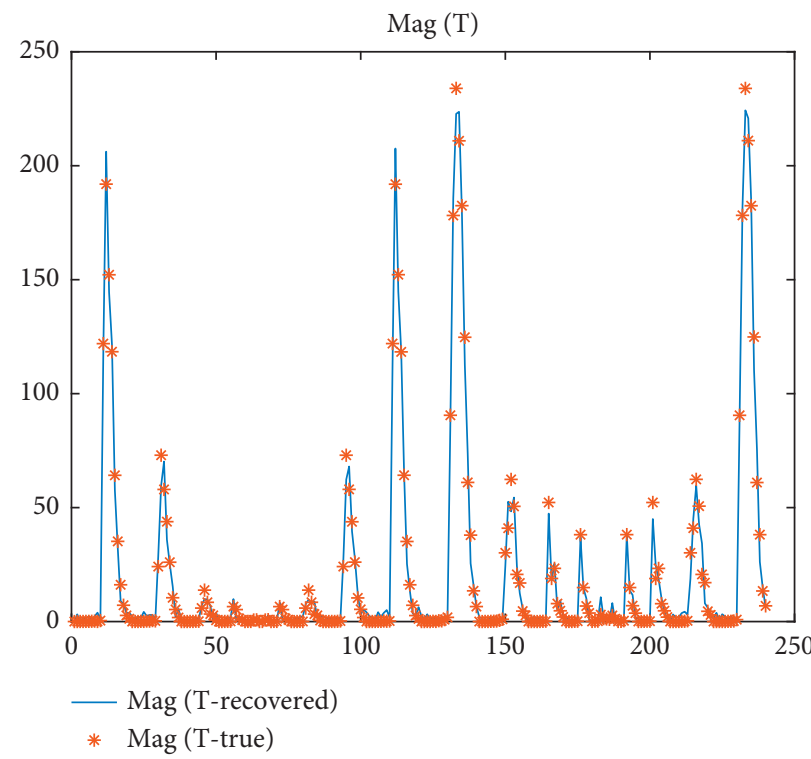

FIGURE 14: The magnitude distribution of the recovered SMC by using the proposed algorithm.

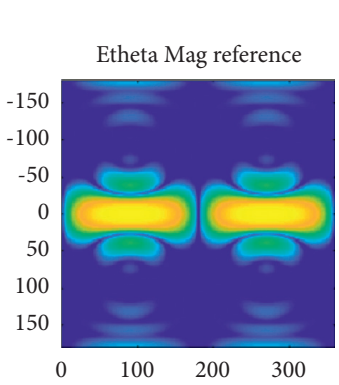

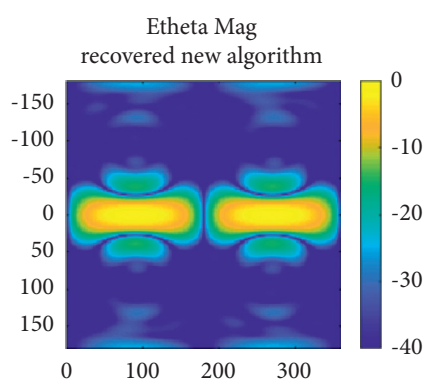

(a)

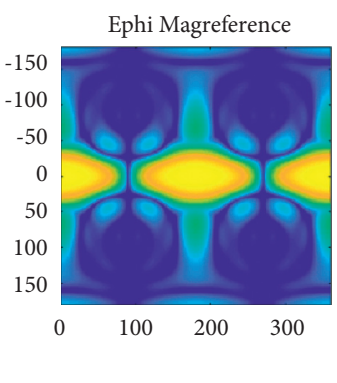

(b)

Figure 15: The reconstructed $E_{\theta}$ and $E_{\varphi}$ of dipole array by adopting the proposed algorithm. (a) $E_{\theta}$. (b) $E_{\varphi}$. 


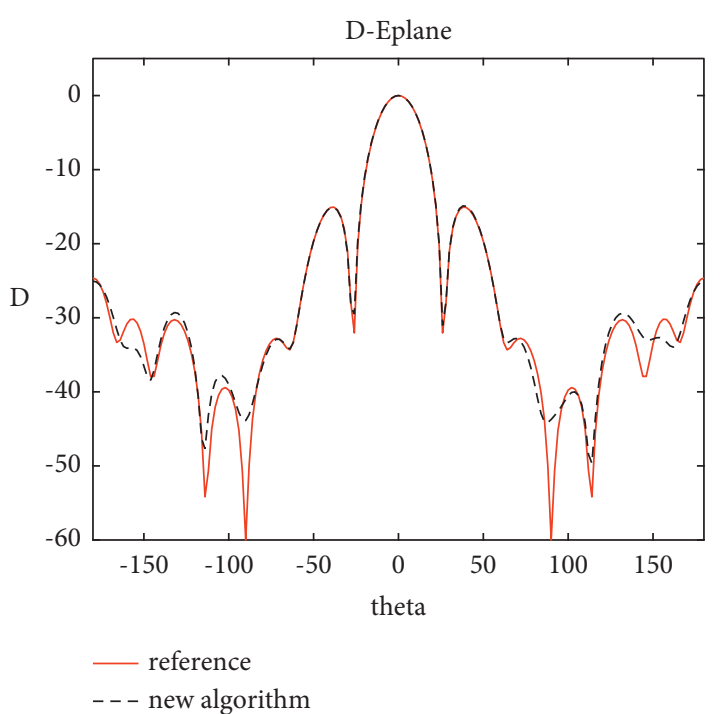

(a)

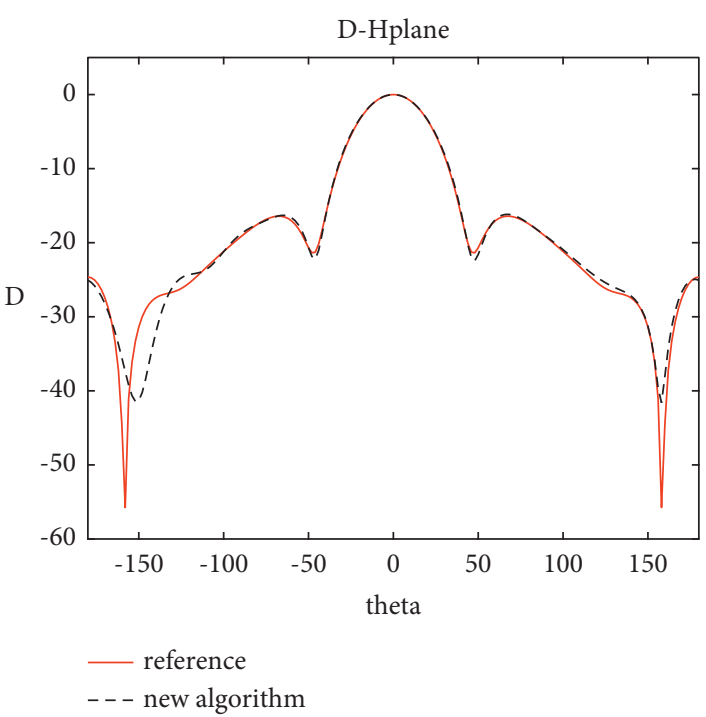

(b)

Figure 16: The obtained radiation pattern of dipole array by adopting the proposed algorithm. (a) E-plane. (b) H-plane.

TABLE 4: The maximum error of extrapolated far field and runtime of the recovery algorithm.

\begin{tabular}{lccc}
\hline Algorithm & Maximum error of H-plane $(\mathrm{dB})$ & Maximum error of E-plane $(\mathrm{dB})$ & Runtime $(\mathrm{s})$ \\
\hline New algorithm & -32.5707 & -38.9251 & 1070.279 \\
G-S & -4.0723 & -10.7640 & 815.339 \\
Wirtinger flow & -3.0923 & -14.1287 & 18.117 \\
Reweighted Wirtinger flow & -3.2298 & -15.7409 & 78.427 \\
Amplitude flow & -4.3138 & -11.2633 & 5.963 \\
Truncated amplitude flow & -5.0701 & -12.0441 & 33.959 \\
Reweighted amplitude flow & -4.5873 & -11.4571 & 10.795 \\
Phase Lamp & -7.1219 & -11.7437 & 3609.99 \\
\hline
\end{tabular}

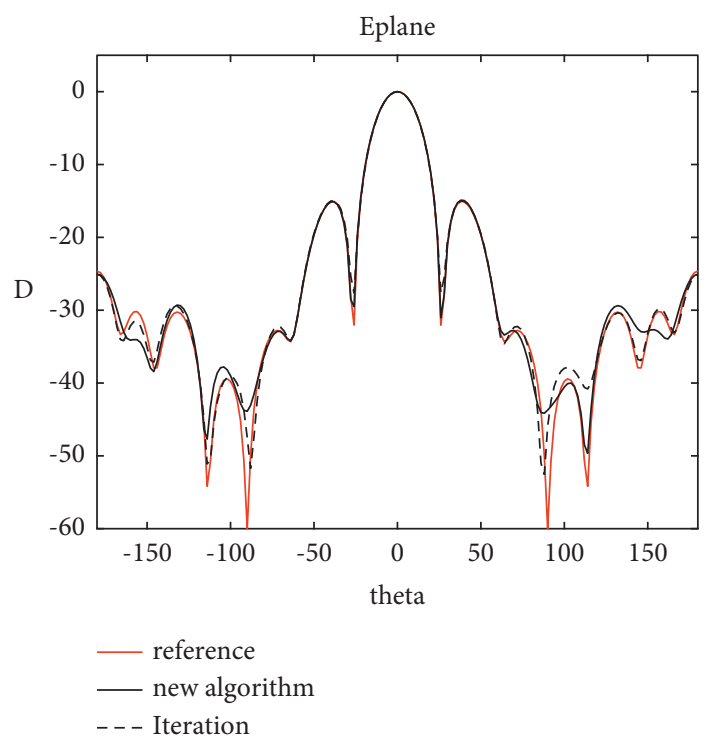

(a)

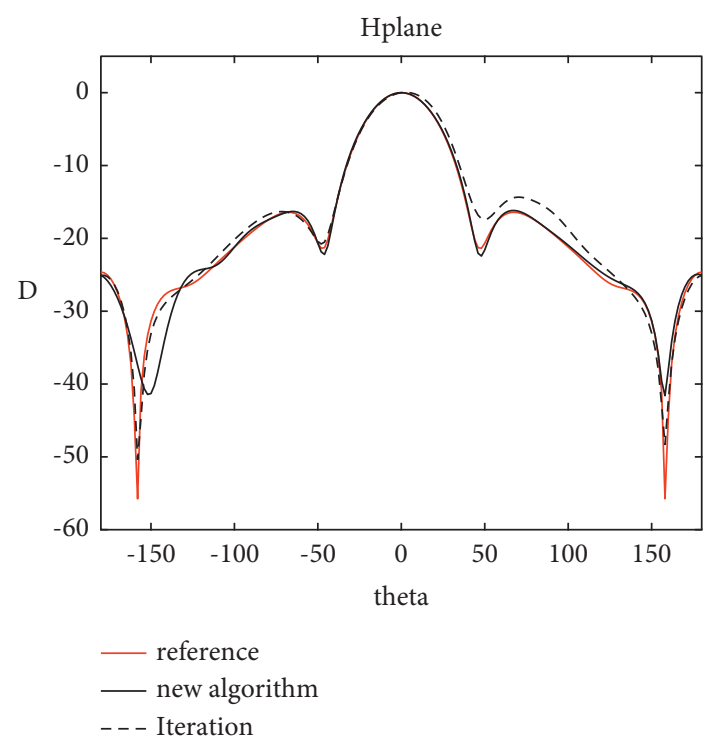

(b)

FIGURE 17: The extrapolated radiation pattern utilizing the algorithm proposed in $[6,8,35]$. (a) E-plane. (b) H-plane. 
TABLE 5: The required near-field sampling points, runtime, and accuracy of the algorithm proposed in this study along with the method in $[6,8,35]$.

\begin{tabular}{lcccc}
\hline Algorithm & Required sampling points & Maximum error of H-plane/dB & Maximum error of E-plane/dB & Runtime (s) \\
\hline New algorithm & 600 & -32.5707 & -38.9251 & 1070.279 \\
Iteration & 7381 & -24.7977 & -28.7271 & 13074 \\
\hline
\end{tabular}
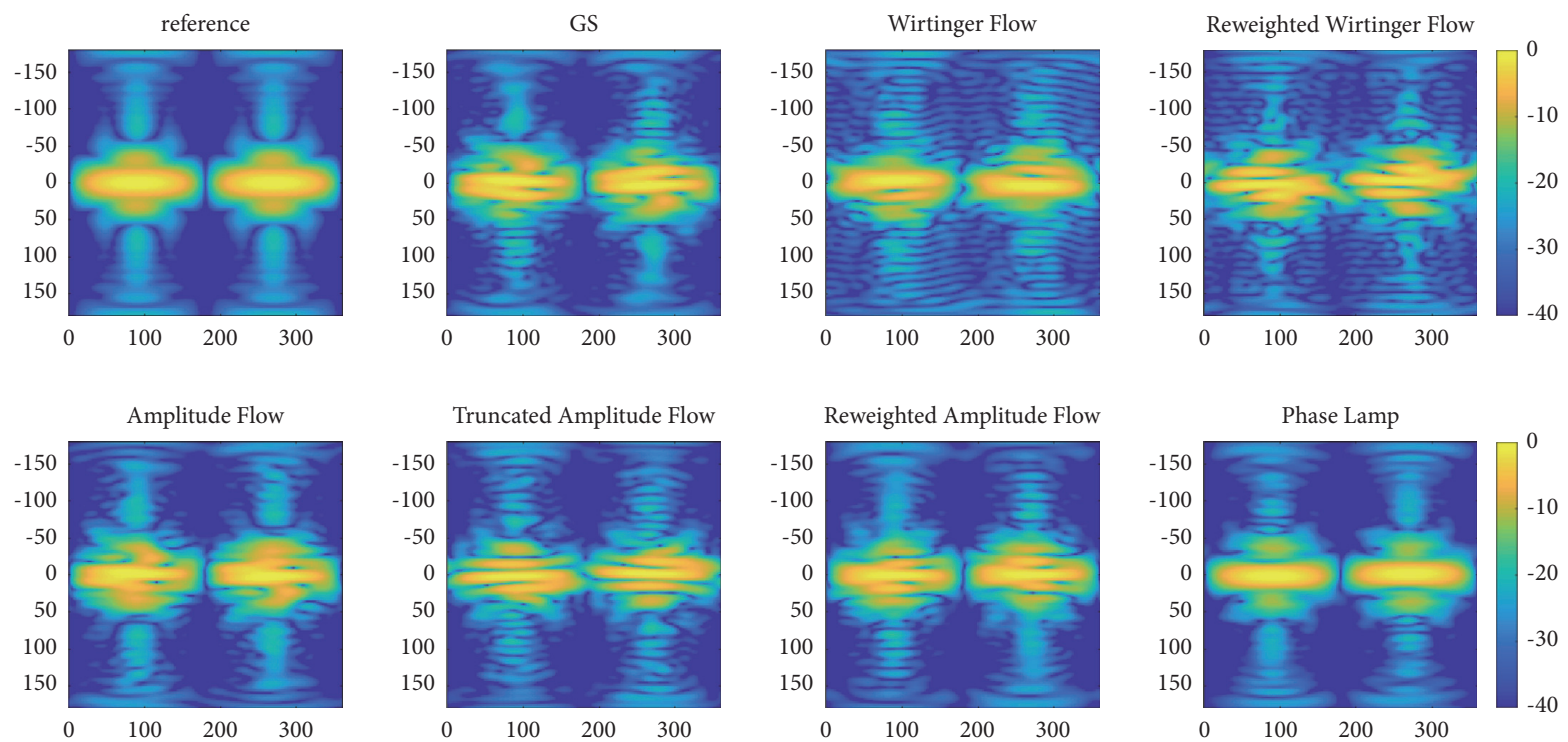

Figure 18: The reconstructed $E_{\theta}$ of horn antenna by adopting other phase recovery algorithms.
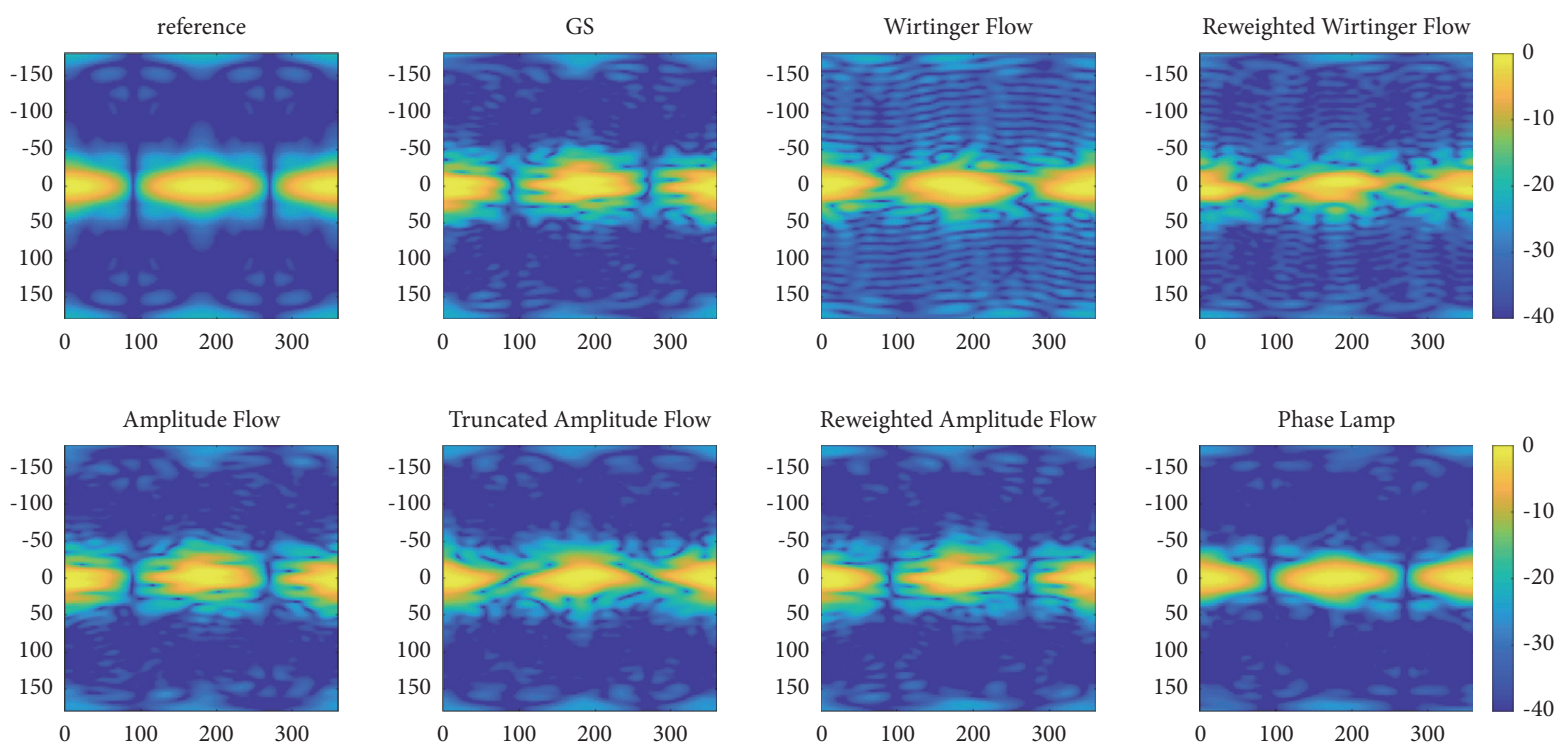

FIgURE 19: The reconstructed $E_{\varphi}$ of horn antenna by adopting other phase recovery algorithms. 


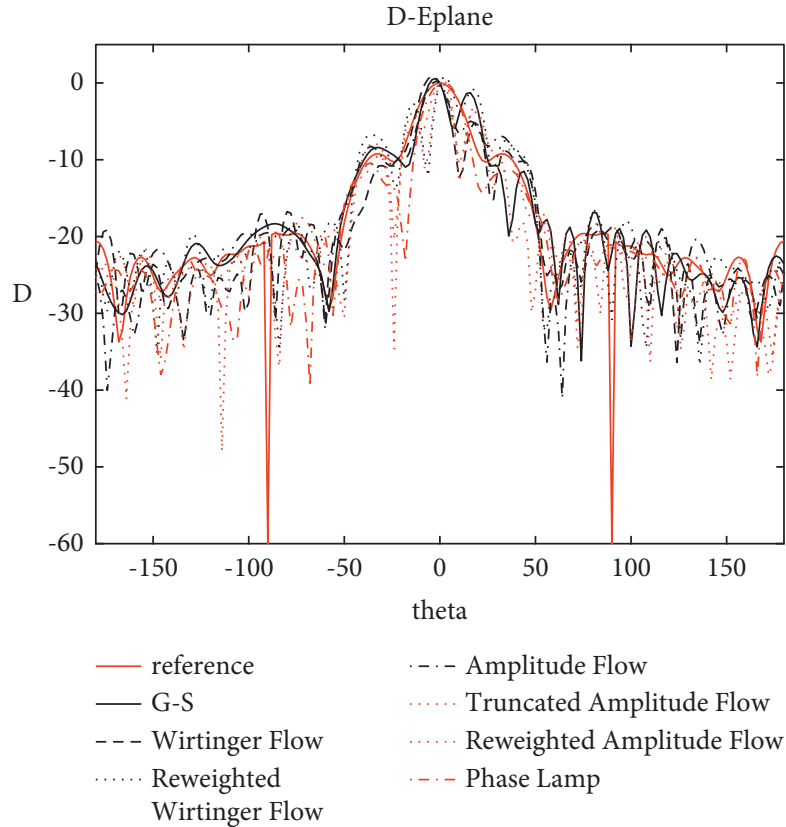

(a)

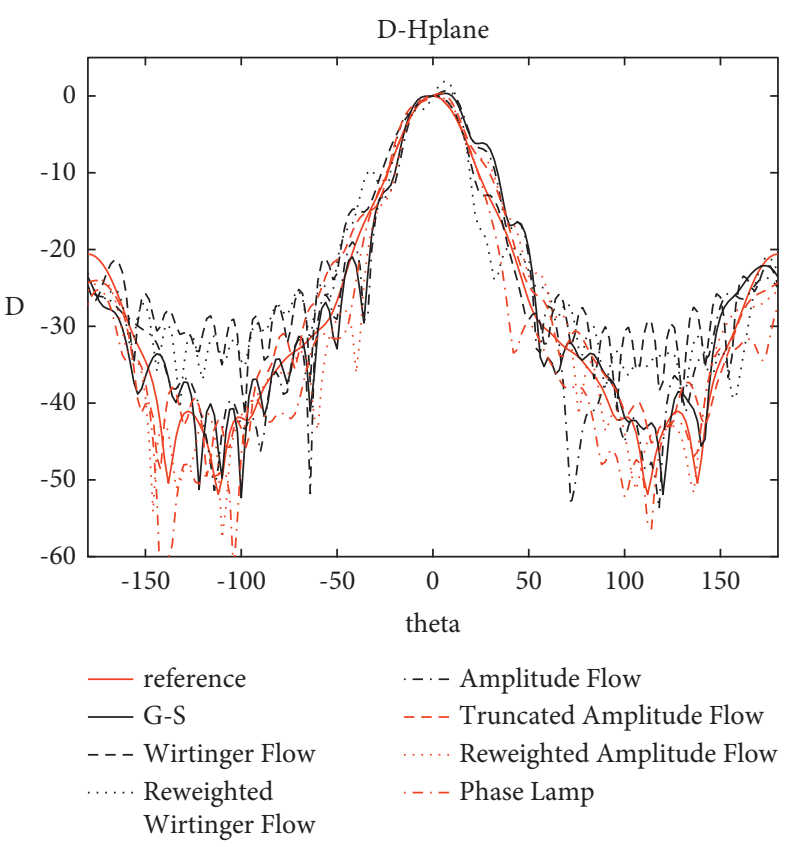

(b)

FIgURE 20: The obtained radiation pattern of horn antenna by adopting other phase recovery algorithms. (a) E-plane. (b) H-plane.

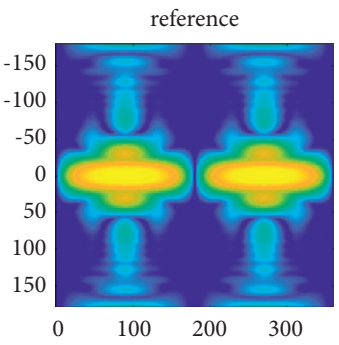

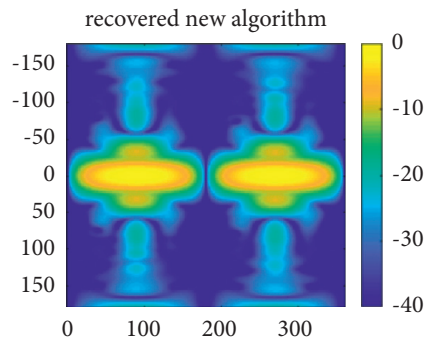

(a)
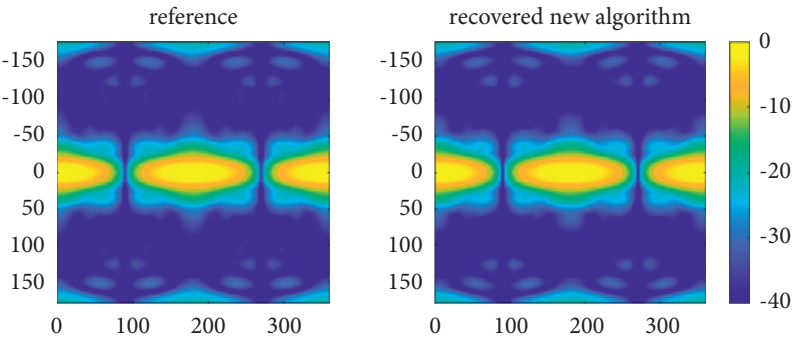

(b)

FIGURE 21: The reconstructed $E_{\theta}$ and $E_{\varphi}$ of horn antenna by adopting the proposed algorithm. (a) $E_{\theta}$. (b) $E_{\varphi}$.

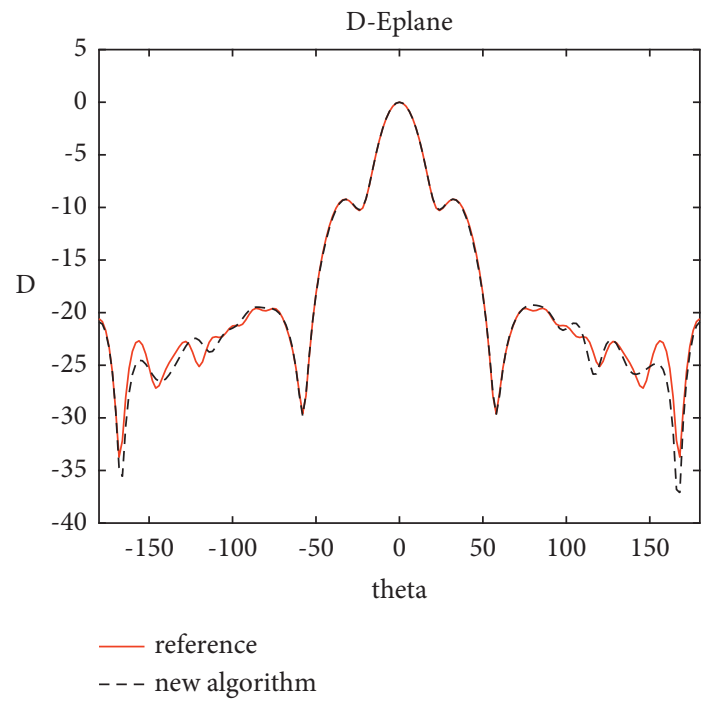

(a)

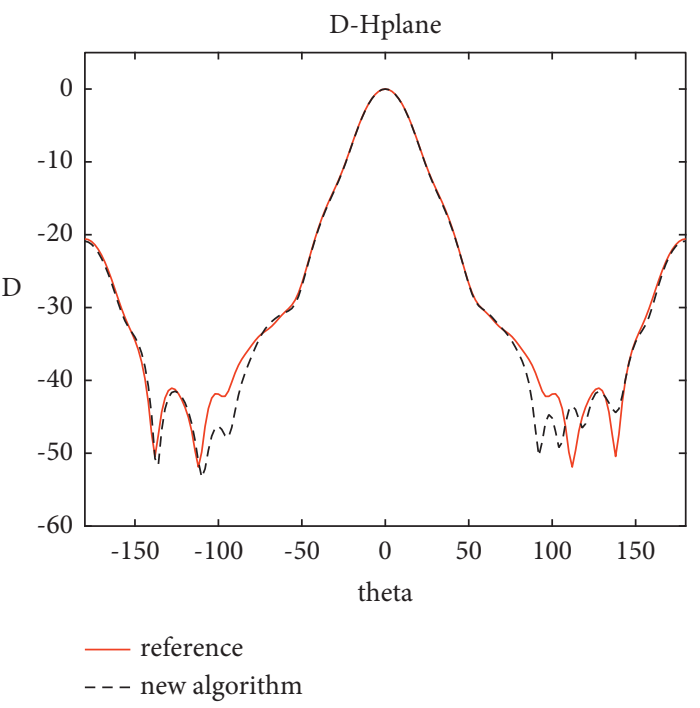

(b)

Figure 22: The obtained radiation pattern of horn antenna by adopting the proposed algorithm. (a) E-plane. (b) H-plane. 
phase recovery algorithms displayed in Figures 18-20, the proposed algorithm exhibits stronger capacity in recovering SMC. Therefore, high precision radiation characteristics of AUT can be acquired, as demonstrated in Figures 21 and 22.

\section{Conclusions}

The proposed algorithm is able to search for the sparsest solution from the feasible domain at a lower oversampling rate and still behaves well for sparse vectors containing more nonzero elements. Besides, the algorithm concentrates on recovering the complex signals without a priori information about the sparsity level. When applied to the spherical NFFFT problem, it can provide a more accurate recovery of SMC, enabling the acquisition of a high-precision radiation pattern. However, the proposed algorithm is converted to solve multiple suboptimization problems, thus running less efficiently, rendering no significant improvement for recovering longer vectors.

Since the near-field sampling strategy indirectly impacts the recovery capability of the proposed algorithm, a more effective sampling method is determined. The quantity of the spheres, the distribution of the measurement points, the radius difference, and polarization are separately investigated by comparing the transformation accuracy and the correlation of the measurement matrix. Numerical results reveal that the measurement matrix corresponding to the two-sphere strategy has a lower correlation, which can compensate for the missing information inherent in phaseless measurements. In particular, with the separation of the two spheres, the field amplitude more significantly varies, and the maximum correlation of the measurement matrix decreases, which can contribute to a higher transformation accuracy. When the sampling points are distributed in the form of a spiral on each sphere, except for the capability to gain accurate radiation characteristics, the measurement trajectory of the positioning device also presents lower complexity, making it more preferable for spherical near-field measurements. Furthermore, the polarization of the probe is also considered in this study. Acquiring two orthogonal polarizations at each sampling point can stably reconstruct the far field of AUT.

\section{Data Availability}

No data were used in this study.

\section{Conflicts of Interest}

The authors declare that they have no conflicts of interest.

\section{Acknowledgments}

This work was supported by the National Natural Science Foundation of China (U1734203).

\section{References}

[1] A. Yaghjian, "An overview of near-field antenna measurements," IEEE Transactions on Antennas and Propagation, vol. 34, no. 1, pp. 30-45, 1986.
[2] J. E. Hansen, "Spherical near-field antenna measurements," IET, vol. 26, 1988.

[3] O. Breinbjerg, "Spherical near-field antenna measurements the most accurate antenna measurement technique," in Proceedings of the 2016 IEEE International Symposium on Antennas and Propagation (APSURSI), pp. 1019-1020, Fajardo, PR, USA, July 2016.

[4] Z. Li, Z. Wang, and J. Wu, "A high precision holographic phaseless near-field method," in Proceedings of the 2013 7th European Conference on Antennas and Propagation (EuCAP), pp. 3594-3598, Gothenburg, Sweden, April 2013.

[5] A. Paulus, J. Knapp, and T. F. Eibert, "Phaseless near-field farfield transformation utilizing combinations of probe signals," IEEE Transactions on Antennas and Propagation, vol. 65, no. 10, pp. 5492-5502, Oct. 2017.

[6] C. H. Schmidt and Y. Rahmat-Samii, "Phaseless spherical near-field antenna measurements: concept, algorithm and simulation," in Proceedings of the 2009 IEEE Antennas and Propagation Society International Symposium, pp. 1-4, North Charleston, SC, USA, July 2009.

[7] J. Fernandez Alvarez, J. M. Bjørstorp, and O. Breinbjerg, "Spherical phaseless probe-corrected near-field measurements of the DTU-ESA VAST12 reflector antenna," in Proceedings of the 40th Annual Symposium of the Antenna Measurement Techniques Association (2018), Williamsburg, VA, USA, September 2018.

[8] C. H. Schmidt, S. F. Razavi, T. F. Eibert, and Y. Rahmat-Samii, "Phaseless spherical near-field antenna measurements for low and medium gain antennas," Advances in Radio Science, vol. 8, pp. 43-48, Sep. 2010.

[9] A. Bangun, C. Culotta-López, A. Behboodi, R. Mathar, and D. Heberling, "On phaseless spherical near-field antenna measurements," in Proceedings of the 2019 13th European Conference on Antennas and Propagation (EuCAP), pp. 1-5, Krakow, Poland, April 2019.

[10] A. Paulus, J. Knapp, and T. F. Eibert, "Utilizing partial knowledge of phase differences in convex optimization for amplitude-only near-field far-field transformation," in Proceedings of the 2017 11th European Conference on Antennas and Propagation (EUCAP), pp. 3766-3770, Paris, France, March 2017.

[11] F. R. Varela, B. G. Iraguen, M. S. Castaner, J. F. Alvarez, M. Mattes, and O. Breinbjerg, "Combination of spherical and planar scanning for phaseless near-field antenna measurements," in Proceedings of the 2019 Antenna Measurement Techniques Association Symposium (AMTA), pp. 1-6, San Diego, CA, USA, October 2019.

[12] A. Guth, C. Culotta-López, J. Maly, H. Rauhut, and D. Heberling, "Polyhedral sampling structures for phaseless spherical near-field antenna measurements," in Proceedings of the 2020 Antenna Measurement Techniques Association Symposium (AMTA), pp. 1-6, Newport, RI, USA, November 2020.

[13] H. Ohlsson, A. Y. Yang, R. Dong, and S. Sastry, CPRL -- an Extension of Compressive Sensing to the Phase Retrieval Problem, NIPS, 2012.

[14] G. Wang, L. Zhang, G. B. Giannakis, M. Akcakaya, and J. Chen, "Sparse phase retrieval via truncated amplitude flow," IEEE Transactions on Signal Processing, vol. 66, no. 2, pp. 479-491, 2018.

[15] D. Needell and J. A. Tropp, "CoSaMP: iterative signal recovery from incomplete and inaccurate samples," Applied and Computational Harmonic Analysis, vol. 26, no. 3, pp. 301-321, 2009.

[16] Y. X. Yuan, "A new stepsize for the steepest descent method," Journal of Computational Mathematics, vol. 24, pp. 149-156, 2006. 
[17] C. Culotta-López, D. Heberling, A. Bangun, A. Behboodi, and R. Mathar, "A compressed sampling for spherical near-field measurements," in Proceedings of the 2018 AMTA Proceedings, pp. 1-6, Williamsburg, VA, USA, November 2018.

[18] B. Fuchs, L. Le Coq, S. Rondineau, and M. D. Migliore, "Compressive sensing approach for fast antenna far field characterization," in Proceedings of the 12th European Conference on Antennas and Propagation (EuCAP 2018), pp. 1-5, London, UK, April 2018.

[19] D. Loschenbrand and C. Mecklenbrauker, "Fast antenna characterization via a sparse spherical multipole expansion," in Proceedings of the 2016 4th International Workshop on Compressed Sensing Theory and its Applications to Radar, Sonar and Remote Sensing (CoSeRa), pp. 212-216, Aachen, Germany, September 2016.

[20] C. Culotta-Lopez, B. Walkenhorst, Q. Ton, and D. Heberling, "Practical considerations in compressed spherical near-field measurements," in Proceedings of the 2019 Antenna Measurement Techniques Association Symposium (AMTA), pp. 1-6, San Diego, CA, USA, October 2019.

[21] Z. Yang, C. Zhang, and L. Xie, "Robust compressive phase retrieval via $\mathrm{L} 1 \mathrm{minimization}$ with application to image reconstruction," 2013, https://arxiv.org/abs/1302.0081.

[22] M. Carlin, P. Rocca, G. Oliveri, F. Viani, and A. Massa, "Directions-of-Arrival estimation through bayesian compressive sensing strategies," IEEE Transactions on Antennas and Propagation, vol. 61, no. 7, pp. 3828-3838, July 2013.

[23] L. Zhang, Y. Lu, B. Wang, F. Li, and Z. Zhang, "Sparse autoencoder with smoothed \$\$1_1\$ 11 regularization," Neural Processing Letters, vol. 47, no. 3, pp. 829-839, 2018.

[24] G. Wang, G. B. Giannakis, and Y. C. Eldar, "Solving systems of random quadratic equations via truncated amplitude flow," IEEE Transactions on Information Theory, vol. 64, no. 2, pp. 773-794, Feb. 2018.

[25] E. J. Candes, X. Li, and M. Soltanolkotabi, "Phase retrieval via wirtinger flow: theory and algorithms," IEEE Transactions on Information Theory, vol. 61, no. 4, pp. 1985-2007, April 2015.

[26] W. Luo, W. Alghamdi, and Y. M. Lu, "Optimal spectral initialization for signal recovery with applications to phase retrieval," IEEE Transactions on Signal Processing, vol. 67, no. 9, pp. 2347-2356, 2019.

[27] R. Cornelius, D. Heberling, N. Koep, A. Behboodi, and R. Mathar, "Compressed sensing applied to spherical nearfield to far-field transformation," in Proceedings of the 2016 10th European Conference on Antennas and Propagation (EuCAP), pp. 1-4, Davos, Switzerland, April 2016.

[28] R. Chandra, Z. Zhong, J. Hontz, V. McCulloch, C. Studer, and T. Goldstein, "Phasepack: a phase retrieval library," vol. 1711, 2017 https://arxiv.org/abs/1711.10175.

[29] G. Wang, G. B. Giannakis, Y. Saad, and J. Chen, "Phase retrieval via reweighted amplitude flow," IEEE Transactions on Signal Processing, vol. 66, no. 11, p. 1, 2018.

[30] Z. Yuan and H. Wang, "Phase retrieval via reweighted Wirtinger flow," Applied Optics, vol. 56, no. 9, p. 2418, 2017.

[31] J. R. Fienup, "Phase retrieval algorithms: a comparison," Applied Optics, vol. 21, p. 15, 1982.

[32] E. B. Saff, A. B. J. Kuijlaars, and B. J. Kuijlaars, "Distributing many points on a sphere," The Mathematical Intelligencer, vol. 19, no. 1, pp. 5-11, 1997.

[33] D. P. Hardin, T. J. Michaels, and E. B. Saff, "A comparison of popular point configurations on S2," 2016, https://arxiv.org/ abs/1607.04590.

[34] R. Cornelius and D. Heberling, "Analysis of sampling grids for spherical near-field antenna measurements," in Proceedings of the Progress In Electromagnetics Research Symposium, Prague, Czech Republic, July 2015.

[35] F. Rodriguez Varela, J. Fernandez Alvarez, B. Galocha Iraguen, M. Sierra Castaner, and O. Breinbjerg, "Numerical and experimental investigation of phaseless spherical near-field antenna measurements," IEEE Transactions on Antennas and Propagation, vol. 69, no. 12, pp. 8830-8841, 2021. 